\title{
EPIDEMIC SPREAD ON ONE-WAY CIRCULAR-COUPLED NETWORKS*
}

\author{
Zhongpu XU (徐忠朴) Xinchu FU (傅新楚) ${ }^{\dagger}$ \\ Department of Mathematics, Shanghai University, Shanghai 200444, China \\ E-mail:xzp0801@163.com; xcfu@shu.edu.cn
}

\begin{abstract}
Real epidemic spreading networks are often composed of several kinds of complex networks interconnected with each other, such as Lyme disease, and the interrelated networks may have different topologies and epidemic dynamics. Moreover, most human infectious diseases are derived from animals, and zoonotic infections always spread on directed interconnected networks. So, in this article, we consider the epidemic dynamics of zoonotic infections on a unidirectional circular-coupled network. Here, we construct two unidirectional three-layer circular interactive networks, one model has direct contact between interactive networks, the other model describes diseases transmitted through vectors between interactive networks, which are established by introducing the heterogeneous mean-field approach method. Then we obtain the basic reproduction numbers and stability of equilibria of the two models. Through mathematical analysis and numerical simulations, it is found that basic reproduction numbers of the models depend on the infection rates, infection periods, average degrees, and degree ratios. Numerical simulations illustrate and expand these theoretical results very well.
\end{abstract}

Key words epidemic dynamics; coupled network; Lyme disease; infective medium; basic reproduction number

2010 MR Subject Classification 92D30; 05C82; 34D23

\section{Introduction}

Infectious diseases have been a major threat to survival and development of human beings. In recent years, the emergence of new infectious diseases have caused much damage to humans, such as H7N9, SARS, Ebola, and Zika, which spread around the world and take the lives of thousands of people [1-3]. So, the mathematical research of epidemic models, epidemic dynamics, prevention and control measures is an important issue, and has attracted great attentions, one of the classic epidemic models was introduced in 1927 [4].

In mathematical epidemiology, the most important is the feasible and practical of mathematical models for various types of infections, only in this way we can provide a better strategy for the prevention and control of diseases in real life. Since the emergence of complex network

${ }^{*}$ Received March 15, 2018; revised August 13, 2018. This work was supported by the National Natural Science Foundation of China (11572181, 11331009).

${ }^{\dagger}$ Corresponding author: Xinchu FU. 
theory $[5,6]$, complex networks have become a powerful tool in studying epidemiology models [7-11]. Especially, a population can be viewed as a complex network, in the sense that a node denotes an individual and a link (edge) denotes the correlation between two individuals, then the disease can spread along edges of the network. In an epidemiology model, because the complexity of different contact types between individuals, it is unreasonable to simplify the complex network to a homogeneous network [12]. And, in order to study the effect of network structures on epidemic spread, many heterogeneous epidemic models have been established [13-15].

Many previous studies focus on a single network, however, real networks are usually large and complex, such as social networks, neural networks, information networks and transportation networks [16-23], and many diseases spread among different populations with various contact patterns. Therefore, it is essential to study epidemic transmission on multiplex networks and coupled networks. Recently, some researchers have achieved important results, which lead to a deeper study on epidemiology [24-30]. For instance, Granell [24] studied a coupled multiplex network embed individual awareness and infection. In [25], the authors discussed the epidemic dynamics of infectious diseases on two interconnected complex networks. In [29], Wang compared network-based approach with homogeneous-mixing approach, then they showed the differences between two methods, and described individual behaviors of epidemic dynamics on complex networks.

In multiplex networks, many studies are based on two-layer interdependent networks, which just contain two populations. In [31], the authors established a mathematical model in a one-way-coupled network with two subnetworks by mean-field approximation approach, then studied the epidemic dynamics of some zoonotic diseases. However, many real epidemics can spread among three populations, the contact pattern and infection rate in each population may be different. For instance, both HBV and HIV can spread among men, women and children. Schistosomiasis spreads via the contacts with patients' skin and infected water, the source of the infection is the patient's feces, so humans and vertebrates are easily infected with Schistosomiasis. Hence, the study of epidemic spreading on three populations has practical and important significance.

Lyme disease, an emerging zoonotic disease of natural nidus caused by Borrelia burgdorferi, which comes from the biting of ticks [32,33]. Over 30000 human beings and animals get infected with Borrelia in America each year. In China, the morbidity of the disease has also been rising in recent years. Previous studies have revealed the transmission of pathogen in three different stages, and explored prevalence of the Lyme pathogen, then found the prevention and control strategy [34-36]. At one stage of the transmission of Lyme disease, the spread of disease between pathogens and susceptible vertebrate hosts may form a cycle. Based on these observations, we first establish an epidemic spreading model on a one-way three-layer circular-coupled network, in which three subnetworks denote three different populations, and the spread of disease is a one-way circular process.

As a matter of fact, the latest surveys reveal that a majority of human pathogens are zoonotic, or caused by animals, such as Black death, Rabies and Spanish influenza [37, 38]. Many infectious diseases transmit in human communities through vectors (infective media). For example, Malaria is a serious disease caused by parasites, which can be transmitted through the biting of infected mosquitoes, and the Black death is a deadly epidemic which spreads by 
rat fleas. Recently, some researches about human-animal coupled epidemic model with infective media on complex networks have drawn attentions [39-42]. Especially, Shi [39] set up a new SIS model with infective medium that describes the epidemic dynamics of vector borne diseases on complex networks. Wang [40] studied an SIS model with an infective vector on complex networks. The disease spreads in two ways, one is direct contact with the infected individuals, the other one is by the vector. In [42], the authors established a time-evolution epidemic model of some zoonotic infections by the mean-field approximation, and analyzed the global dynamics.

Some previous researches are mainly about epidemic models with an infective medium on an interconnected network, which contains two separated networks of populations (humans or animals). Here, in this article, we consider a one-way three-layer circular-coupled network with three different vectors (infective media), in which between any two subnetworks has an infective medium. The study of this special epidemic model will provide a new insight into the zoonotic infections on interconnected networks.

The rest of the article is arranged as follows. In Section 2, a directed three-layer circularcoupled network is introduced by heterogeneous mean-field approach method, and the basic reproduction number is estimated, then the global stability analysis of equilibria of the system is given. In Section 3, a directed three-layer circular-coupled network with three infective media is considered. Similarly, the basic reproduction number is calculated, and the global dynamics of the system is presented. In Section 4, some numerical simulations to illustrate and complement the theoretical results of the two models are performed. Finally, conclusions and discussions are given in Section 5 .

\section{One-way Circular-coupled Network}

In this section, we consider a directed circular-coupled network model. The whole network includes three subnetworks, $A, B$ and $C$, and there are cross contacts among three subnetworks, that is, the nodes in $A, B$ and $C$ all have three types of degrees. Moreover, the disease spreading in three subnetworks is one-way, which means the nodes in $A$ can infect the nodes in $B$, similarly, subnetwork $B$ can infect $C$, subnetwork $C$ can infect $A$, but not the reverse. A graphical representation of the network is described in Fig. 1.

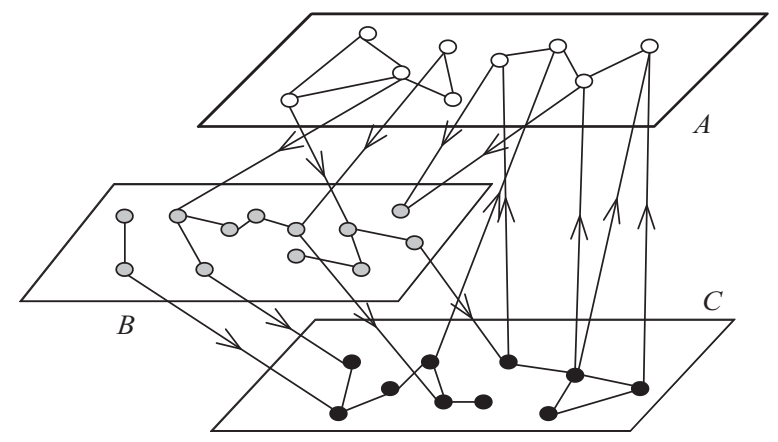

Figure 1 A one-way circular-coupled network with three subnetworks

In each subnetwork, we consider the classical SIS epidemic model, so each individual can be in two opposing states: susceptible (S) and infected (I). To describe the epidemic spreading 
process of the whole network, we let $P_{A}(i, j, k)$ be the probability of a random node in $A$ with $i$ links within $A, j$ links connecting $A$ and $B$, and $k$ links connecting $A$ and $C, P_{B}(i, j, k)$ and $P_{C}(i, j, k)$ are similarly defined. And, other parameters in the network are given in Table 1.

Table 1 Definition of the network parameters

\begin{tabular}{cc}
\hline Parameter & Definition $(X=A, B$ or $C)$ \\
\hline$N_{i, j, k}^{X}$ & Number of nodes in $X$ with $(i, j, k)$-degree \\
$S_{i, j, k}^{X}\left(\right.$ or $\left.I_{i, j, k}^{X}\right)$ & Number of susceptible (infected) nodes in $X$ with $(i, j, k)$-degree \\
$n_{11}\left(\right.$ or $n_{12}$ or $\left.n_{13}\right)$ & Maximum degree of nodes in $A$ connecting $A$ (or $B$ or $C$ ) \\
$n_{21}\left(\right.$ or $n_{22}$ or $\left.n_{23}\right)$ & Maximum degree of nodes in $B$ connecting $A$ (or $B$ or $C$ ) \\
$n_{31}\left(\right.$ or $n_{32}$ or $\left.n_{33}\right)$ & Maximum degree of nodes in $C$ connecting $A$ (or $B$ or $C$ ) \\
$\langle k\rangle_{11}\left(\right.$ or $\langle k\rangle_{12}$ or $\left.\langle k\rangle_{13}\right)$ & Average degree of nodes in $A$ connecting $A$ (or $B$ or $C$ ) \\
$\langle k\rangle_{21}\left(\right.$ or $\langle k\rangle_{22}$ or $\left.\langle k\rangle_{23}\right)$ & Average degree of nodes in $B$ connecting $A$ (or $B$ or $C$ ) \\
$\langle k\rangle_{31}\left(\right.$ or $\langle k\rangle_{32}$ or $\left.\langle k\rangle_{33}\right)$ & Average degree of nodes in $C$ connecting $A$ (or $B$ or $C)$ \\
\hline
\end{tabular}

According to Table 1, we can obtain the total number of susceptible nodes, infected nodes and all nodes in subnetwork $A$, respectively, that is,

$$
S^{A}=\sum_{i=0}^{n_{11}} \sum_{j=0}^{n_{12}} \sum_{k=0}^{n_{13}} S_{i, j, k}^{A}, I^{A}=\sum_{i=0}^{n_{11}} \sum_{j=0}^{n_{12}} \sum_{k=0}^{n_{13}} I_{i, j, k}^{A}, N^{A}=S^{A}+I^{A},
$$

the subnetworks $B$ and $C$ are similar.

The joint degree distributions are

$$
P_{A}(i, j, k)=\frac{N_{i, j, k}^{A}}{N^{A}}, P_{B}(i, j, k)=\frac{N_{i, j, k}^{B}}{N^{B}}, P_{C}(i, j, k)=\frac{N_{i, j, k}^{C}}{N^{C}}
$$

the marginal degree distributions are

$$
P_{A}(i, \cdot, \cdot)=\sum_{j=0}^{n_{12}} \sum_{k=0}^{n_{13}} P_{A}(i, j, k), P_{A}(\cdot, j, \cdot)=\sum_{i=0}^{n_{11}} \sum_{k=0}^{n_{13}} P_{A}(i, j, k), P_{A}(\cdot, \cdot, k)=\sum_{i=0}^{n_{11}} \sum_{j=0}^{n_{12}} P_{A}(i, j, k),
$$

the average degree values are

$$
\langle k\rangle_{11}=\sum_{i=0}^{n_{11}} i P_{A}(i, \cdot, \cdot),\langle k\rangle_{12}=\sum_{j=0}^{n_{12}} j P_{A}(\cdot, j, \cdot),\langle k\rangle_{13}=\sum_{k=0}^{n_{13}} k P_{A}(\cdot, \cdot, k),
$$

and the second moments of degree are

$$
\left\langle k^{2}\right\rangle_{11}=\sum_{i=0}^{n_{11}} i^{2} P_{A}(i, \cdot, \cdot),\left\langle k^{2}\right\rangle_{12}=\sum_{j=0}^{n_{12}} j^{2} P_{A}(\cdot, j, \cdot),\left\langle k^{2}\right\rangle_{13}=\sum_{k=0}^{n_{13}} k^{2} P_{A}(\cdot, \cdot, k),
$$

the subnetworks $B$ and $C$ are similar.

For simplicity, we consider the whole model as static, so the sizes $N^{A}, N^{B}$ and $N^{C}$ are all constant. In the model, a susceptible node in subnetwork $A$ (or $B$ or $C$ ) can be infected with rate $\lambda_{11}$ (or $\lambda_{22}$ or $\lambda_{33}$ ) if it is contacted with an infected node in subnetwork $A$ (or $B$ or $C$ ), respectively. A susceptible node in subnetwork $B$ has probability $\lambda_{12}$ of contagion with an infected node in subnetwork $A$, a susceptible node in subnetwork $C$ can be infected with probability $\lambda_{23}$ if it is contacted with an infected node in subnetwork $B$, and the probability $\lambda_{31}$ of a susceptible node in subnetwork $A$ can be infected by an infected node in subnetwork 
$C$. Each infected node in subnetworks $A, B$ and $C$ becomes susceptible with rate $\mu_{1}, \mu_{2}$ and $\mu_{3}$, respectively.

Let $s_{e, g, h}^{A}=\frac{S_{e, g, h}^{A}}{N_{e, g, h}^{A},}, \rho_{e, g, h}^{A}=\frac{I_{e, g, h}^{A}}{N_{e, g, h}^{A}}$ as the corresponding densities of subnetwork $A$, similar to the definition of $s_{l, m, n}^{B}, \rho_{l, m, n}^{B}, s_{w, x, z}^{C}$, and $\rho_{w, x, z}^{C}$. For simplicity, we suppose that the degree is uncorrelated in the network, that is, the connectivity of any node is uncorrelated to the connectivity of its neighbors. Then we give the dynamical mean-field reaction rate equations of the epidemic network, which is composed of $\left[\left(n_{11}+1\right)\left(n_{12}+1\right)\left(n_{13}+1\right)+\left(n_{21}+1\right)\left(n_{22}+\right.\right.$ 1) $\left.\left(n_{23}+1\right)+\left(n_{31}+1\right)\left(n_{32}+1\right)\left(n_{33}+1\right)\right]$-dimensional ordinary differential equations:

$$
\left\{\begin{array}{l}
\frac{\mathrm{d} \rho_{e, g, h}^{A}(t)}{\mathrm{d} t}=\lambda_{11} e\left(1-\rho_{e, g, h}^{A}(t)\right) \Theta_{11}(t)+\lambda_{31} h\left(1-\rho_{e, g, h}^{A}(t)\right) \Theta_{31}(t)-\mu_{1} \rho_{e, g, h}^{A}(t), \\
\frac{\mathrm{d} \rho_{l, m, n}^{B}(t)}{\mathrm{d} t}=\lambda_{12} l\left(1-\rho_{l, m, n}^{B}(t)\right) \Theta_{12}(t)+\lambda_{22} m\left(1-\rho_{l, m, n}^{B}(t)\right) \Theta_{22}(t)-\mu_{2} \rho_{l, m, n}^{B}(t), \\
\frac{\mathrm{d} \rho_{w, x, z}^{C}(t)}{\mathrm{d} t}=\lambda_{23} x\left(1-\rho_{w, x, z}^{C}(t)\right) \Theta_{23}(t)+\lambda_{33} z\left(1-\rho_{w, x, z}^{C}(t)\right) \Theta_{33}(t)-\mu_{3} \rho_{w, x, z}^{C}(t),
\end{array}\right.
$$

where $e=0,1, \cdots, n_{11}, g=0,1, \cdots, n_{12}, h=0,1, \cdots, n_{13}, l=0,1, \cdots, n_{21}, m=0,1, \cdots, n_{22}$, $n=0,1, \cdots, n_{23}, w=0,1, \cdots, n_{31}, x=0,1, \cdots, n_{32}, z=0,1, \cdots, n_{33}$, and the meanings of $\Theta_{i j}$ for the situation $(i, j)=(1,1),(1,2),(2,2),(2,3),(3,1)$ and $(3,3)$ are as follows

$$
\begin{aligned}
& \Theta_{11}(t)=\frac{1}{\langle k\rangle_{11}} \sum_{i=0}^{n_{11}} \sum_{j=0}^{n_{12}} \sum_{k=0}^{n_{13}} i P_{A}(i, j, k) \rho_{i, j, k}^{A}(t), \Theta_{12}(t)=\frac{1}{\langle k\rangle_{12}} \sum_{i=0}^{n_{11}} \sum_{j=0}^{n_{12}} \sum_{k=0}^{n_{13}} j P_{A}(i, j, k) \rho_{i, j, k}^{A}(t), \\
& \Theta_{22}(t)=\frac{1}{\langle k\rangle_{22}} \sum_{i=0}^{n_{21}} \sum_{j=0}^{n_{22}} \sum_{k=0}^{n_{23}} j P_{B}(i, j, k) \rho_{i, j, k}^{B}(t), \Theta_{23}(t)=\frac{1}{\langle k\rangle_{23}} \sum_{i=0}^{n_{21}} \sum_{j=0}^{n_{22}} \sum_{k=0}^{n_{23}} k P_{B}(i, j, k) \rho_{i, j, k}^{B}(t), \\
& \Theta_{31}(t)=\frac{1}{\langle k\rangle_{31}} \sum_{i=0}^{n_{31}} \sum_{j=0}^{n_{32}} \sum_{k=0}^{n_{33}} i P_{C}(i, j, k) \rho_{i, j, k}^{C}(t), \Theta_{33}(t)=\frac{1}{\langle k\rangle_{33}} \sum_{i=0}^{n_{31}} \sum_{j=0}^{n_{32}} \sum_{k=0}^{n_{33}} k P_{C}(i, j, k) \rho_{i, j, k}^{C}(t) .
\end{aligned}
$$

In the model, these special cases should be considered. (1) Isolated nodes as $(0,0,0)$-degree cannot propagate in the model, and if the nodes are infected at initial state, they will eventually recover at $\mu_{1}, \mu_{2}$ and $\mu_{3}$; (2) If $n_{12}=n_{13}=n_{21}=n_{22}=n_{23}=n_{31}=n_{32}=n_{33}=0$, the model becomes the classical SIS model; (3) If $n_{12}=n_{21}=0$ ( or $n_{23}=n_{32}=0$ or $n_{31}=n_{13}=0$ ), then a one-way circular-coupled network becomes a one-way string-coupled network.

An important epidemiological parameter is basic reproduction number $R_{0}$, which is defined as the expected number of secondary infections produced by a single infective individual in a completely susceptible population.

Hence, we will calculate the basic reproduction number $R_{0}$. Let $\rho_{0,0,0}^{A}=y_{1}, \rho_{0,0,1}^{A}=$ $y_{2}, \cdots, \rho_{n_{11}, n_{12}, n_{13}}^{A}=y_{\left(n_{11}+1\right)\left(n_{12}+1\right)\left(n_{13}+1\right)}, \rho_{0,0,0}^{B}=y_{\left(n_{11}+1\right)\left(n_{12}+1\right)\left(n_{13}+1\right)+1}, \cdots, \rho_{n_{21}, n_{22}, n_{23}}^{B}=$

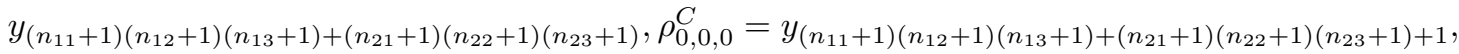
$\cdots, \rho_{n_{31}, n_{32}, n_{33}}^{C}=y_{N}$, where $N=\left[\left(n_{11}+1\right)\left(n_{12}+1\right)\left(n_{13}+1\right)+\left(n_{21}+1\right)\left(n_{22}+1\right)\left(n_{23}+1\right)+\right.$ $\left.\left(n_{31}+1\right)\left(n_{32}+1\right)\left(n_{33}+1\right)\right]$. Then, $f=\left(f_{1}, f_{2}, \cdots, f_{N}\right)$ and $y=\left(y_{1}, y_{2}, \cdots, y_{N}\right)$ are both $N$-dimensional vectors, and $f$ denotes the right hand sides of model (2.1). So, the model is rewritten as

$$
\frac{\mathrm{d} y(t)}{\mathrm{d} t}=f(y(t))
$$

It is easy to know the model has the disease-free equilibrium $E_{0}$ with $y_{i}=0$ for all $i=$ $1,2, \cdots, N$. 
According to [43], the basic reproduction number can be computed by $R_{0}=\rho\left(F V^{-1}\right)$, which represents the spectral radius of matrix $F V^{-1}, F$ is the probability of occurring new infections, and $V$ is the probability of individuals removing from the population. For the model, let $\mathscr{F}$ and $\mathscr{V}$ are

$$
\begin{gathered}
\mathscr{F}=\left(\begin{array}{c}
\lambda_{11} e\left(1-\rho_{e, g, h}^{A}\right) \Theta_{11}+\lambda_{31} h\left(1-\rho_{e, g, h}^{A}\right) \Theta_{31} \\
\lambda_{12} l\left(1-\rho_{l, m, n}^{B}\right) \Theta_{12}+\lambda_{22} m\left(1-\rho_{l, m, n}^{B}\right) \Theta_{22} \\
\lambda_{23} x\left(1-\rho_{w, x, z}^{C}\right) \Theta_{23}+\lambda_{33} z\left(1-\rho_{w, x, z}^{C}\right) \Theta_{33}
\end{array}\right), \\
\mathscr{V}=\left(\begin{array}{c}
\mu_{1} \rho_{e, g, h}^{A} \\
\mu_{2} \rho_{l, m, n}^{B} \\
\mu_{3} \rho_{w, x, z}^{C}
\end{array}\right) .
\end{gathered}
$$

By [43], the matrix $F=\left(\frac{\partial \mathscr{F}_{i}\left(E_{0}\right)}{\partial y_{j}}\right), V=\left(\frac{\partial \mathscr{Y}_{i}\left(E_{0}\right)}{\partial y_{j}}\right)$, where $i, j=0,1, \cdots, N$. Obviously, matrix $V$ is diagonal matrix, where $v_{i i}=\mu_{1}, i=1, \cdots,\left(n_{11}+1\right)\left(n_{12}+1\right)\left(n_{13}+1\right), v_{j j}=\mu_{2}$, $j=\left(n_{11}+1\right)\left(n_{12}+1\right)\left(n_{13}+1\right)+1, \cdots,\left(n_{11}+1\right)\left(n_{12}+1\right)\left(n_{13}+1\right)+\left(n_{21}+1\right)\left(n_{22}+1\right)\left(n_{23}+1\right)$, $v_{k k}=\mu_{3}, k=\left(n_{11}+1\right)\left(n_{12}+1\right)\left(n_{13}+1\right)+\left(n_{21}+1\right)\left(n_{22}+1\right)\left(n_{23}+1\right)+1, \cdots, N$. Hence, we assume that the joint degree distributions are independent, then

$$
P_{X}(i, j, k)=P_{X}(i, \cdot, \cdot) P_{X}(\cdot, j, \cdot) P_{X}(\cdot, \cdot, k), X=A, B, C .
$$

By means of similar transformations, the next-generation matrix $\Gamma=F V^{-1}$ can be rewritten as follows

$$
\Gamma=\left(\begin{array}{cccccc}
\frac{\lambda_{11}\left\langle k^{2}\right\rangle_{11}}{\mu_{1}\langle k\rangle_{11}} & \frac{\lambda_{11}}{\mu_{1}}\langle k\rangle_{13} & 0 & 0 & 0 & 0 \\
0 & 0 & 0 & 0 & \frac{\lambda_{31}}{\mu_{3}}\langle k\rangle_{32} & \frac{\lambda_{31}}{\mu_{3}}\langle k\rangle_{33} \\
\frac{\lambda_{12}}{\mu_{1}}\langle k\rangle_{11} & \frac{\lambda_{12}}{\mu_{1}}\langle k\rangle_{13} & 0 & 0 & 0 & 0 \\
0 & 0 & \frac{\lambda_{22}}{\mu_{2}}\langle k\rangle_{21} \frac{\lambda_{22}\left\langle k^{2}\right\rangle_{22}}{\mu_{2}\langle k\rangle_{22}} & 0 & 0 \\
0 & 0 & \frac{\lambda_{23}}{\mu_{2}}\langle k\rangle_{21} & \frac{\lambda_{23}}{\mu_{2}}\langle k\rangle_{22} & 0 & 0 \\
0 & 0 & 0 & 0 & \frac{\lambda_{33}}{\mu_{3}}\langle k\rangle_{32} \frac{\lambda_{33}\left\langle k^{2}\right\rangle_{33}}{\mu_{3}\langle k\rangle_{33}}
\end{array}\right) .
$$

In the model, the basic reproduction number $R_{0}$ cannot be explicitly expressed. For the nonnegative matrix $\Gamma$, it can be verified directly that $\Gamma$ is irreducible, then by the PerronFrobenius theorem, we know the relationship between $R_{0}$ and infection rates $\lambda$, infection periods $1 / \mu$, average degrees $\langle k\rangle$, and degree ratio $\left\langle k^{2}\right\rangle /\langle k\rangle$. So, we can estimate $R_{0}$ as follows

$$
\min \left\{r_{1}, r_{2}, \cdots, r_{6}\right\} \leq R_{0} \leq \max \left\{r_{1}, r_{2}, \cdots, r_{6}\right\},
$$

where $r_{i}$ is the sum of the elements in the $i$-th row (or column) of matrix (2.5).

From the Theorem 2 of [43], we can obtain the following result.

Theorem 2.1 For the disease transmission model (2.1), the disease-free equilibrium $E_{0}$ is locally asymptotically stable if $R_{0}<1$; otherwise, $E_{0}$ becomes unstable if $R_{0}>1$.

Now, we consider the global stability of the model (2.1). 
Theorem 2.2 For model (2.1), $\Omega \triangleq\left\{y=\left\{y_{1}, y_{2}, \cdots, y_{N}\right\}, 0 \leq y_{i} \leq 1, i=1,2, \cdots, N\right\}$ is a positively invariant set.

Proof We prove the positive invariance of the set $\Omega$ by using the contradiction method. First, we prove that if initial value $y(0) \in \Omega$, then $y_{i}(t) \geq 0$ for all $t>0, i=1,2, \cdots, N$. Otherwise, there are a $l_{0} \in\{1,2, \cdots, N\}$ and $t_{0}>0$, such that $y_{l_{0}}\left(t_{0}\right)=0$. Let $t^{*}=\inf \{t>$ $\left.0, y_{i}(t)=0\right\}$, and we assume $y_{l_{0}}\left(t^{*}\right)=\rho_{i_{0}, j_{0}, k_{0}}^{A}\left(t^{*}\right)=0$, by the definition of $t^{*}$, we have $\mathrm{d} \rho_{i_{0}, j_{0}, k_{0}}^{A}\left(t^{*}\right) / \mathrm{d} t \leq 0$.

However, from model (2.1), it is easy to get $\mathrm{d} \rho_{i_{0}, j_{0}, k_{0}}^{A}\left(t^{*}\right) / \mathrm{d} t=\lambda_{11} i_{0} \Theta_{11}\left(t^{*}\right)+\lambda_{31} k_{0} \Theta_{31}\left(t^{*}\right)>$ 0 , which is a contradiction. So, we obtain $y_{i}(t) \geq 0, i=1, \cdots, N$ for all $t>0$. Because of $s_{i, j, k}^{X}(t)=1-\rho_{i, j, k}^{X}(t)$ for $X=A, B, C$, similarly, we can also verify that $s_{i, j, k}^{X}(t) \geq 0$ for all $t>0$. Then, we obtain $0 \leq \rho_{i, j, k}^{X}(t) \leq 1$. The proof is finished.

Before we discuss global stability of the disease-free equilibrium and endemic equilibrium, Corollary 3.2 in [44] is introduced as the Lemma 2.3 as follows.

Lemma 2.3 ([44]) For the model (2.2), let $f: R_{+}^{n} \rightarrow R^{n}$ be a continuously differentiable map. Assume that

(1) $f$ is cooperative on $R_{+}^{n}$ and $D f(y)=\left(\partial f_{i} / \partial y_{j}\right)_{1 \leq i, j \leq N}$ is irreducible for every $y \in R_{+}^{n}$;

(2) $f(0)=0$ and $f_{i}(y) \geq 0$ for all $y \in R_{+}^{n}$ with $y_{i}=0, i=1,2, \cdots, N$;

(3) $f$ is strictly sublinear on $R_{+}^{n}$, that is, for any $\alpha \in(0,1)$ and any $y \gg 0, f(\alpha y)>\alpha f(y)$.

(a) If $s(D f(0)) \leq 0$, then $y=0$ is globally asymptotically stable with respect to $R_{+}^{n}$;

(b) If $s(D f(0))>0$, then either

(i) for any $y \in R_{+}^{n} \backslash\{\mathbf{0}\}, \lim _{t \rightarrow \infty}|\varphi(t, y)|=+\infty$, or alternatively,

(ii) the model admits a unique positive steady state $y^{*} \gg 0$ and $y=y^{*}$ is globally asymptotically stable with respect to $R_{+}^{n} \backslash\{\mathbf{0}\}$.

Next, we give the following theorem to show the stability of the equilibria.

Theorem 2.4 The disease-free equilibrium $E_{0}$ of model (2.1) is globally asymptotically stable in $\Omega$ when $R_{0} \leq 1$; Otherwise, a unique endemic equilibrium $E^{*}$ exists for the model (2.1), and it is globally asymptotically stable in $\Omega-\{\mathbf{0}\}$ when $R_{0}>1$.

Proof It is easy to show the model (2.2) satisfies the condition (2) of the above lemma, the function $f: \Omega \rightarrow R^{n}$ is continuously differentiable, and $f$ is cooperative on $\Omega$, that is, $\partial f_{i} / \partial g_{j} \geq$ 0 for all $i, j=1,2, \cdots, N$, and $i \neq j$. Furthermore, we know that $D f(y)=\left(\partial f_{i} / \partial y_{i}\right)_{1 \leq i, j \leq N}$ is irreducible for $y \in \Omega$. Moreover, for any $\varepsilon \in(0,1)$ and $y \in \Omega, f_{i}(\varepsilon y) \geq \varepsilon f_{i}(y), i=1,2, \cdots, N$, which implies that $f$ is strictly sublinear in $\Omega$.

By Lemma 2.3, if $R_{0} \leq 1(s(D f(0)) \leq 0)$, then the disease-free equilibrium $E_{0}$ is globally asymptotically stable in $\Omega$, if $R_{0}>1(s(D f(0))>0)$, we know the case (i) is impossible by Theorem 2.2, then there exists a unique endemic equilibrium $E^{*}$ in the model $(2.1)$, which is globally asymptotically stable in $\Omega-\{\mathbf{0}\}$.

\section{One-way Circular-coupled Network with Infective Media}

In this section, we consider a directed circular-coupled network with infective media. The interaction model contains three population networks $A, B$ and $C$, and three vectors $a, b$ and $c$. Assume that there is no direct contact among networks $A, B$ and $C$, the infections are spread 
through the vectors. The direction of propagation is one-way and becomes a cycle network. The network as shown in Fig. 2.

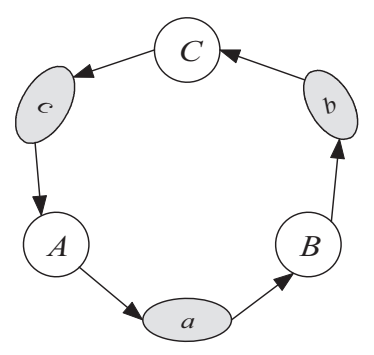

Figure 2 A one-way circular-coupled network with three subnetworks and three vectors

In the model, a susceptible node in subnetwork $A$ (or $B$ or $C$ ) is infected with rate $\lambda_{1}$ (or $\lambda_{2}$ or $\left.\lambda_{3}\right)$ if it is contacted with an infected node in the same subnetwork at each time step. A susceptible node in subnetwork $A$ also can infected with rate $r_{32}$ by contacts with infected vector $c$, and the vectors $a$ and $b$ have no effect on the nodes in subnetwork $A$. Similarly, a susceptible node in subnetwork $B$ (or $C$ ) is infected with rate $r_{12}\left(\right.$ or $\left.r_{22}\right)$ if it contacts with infected vector $a$ (or $b$ ). On the other hand, each susceptible vector $a$ (or $b$ or $c$ ) is infected with probability $r_{11}$ (or $r_{21}$ or $r_{31}$ ) through contacts with infected individuals in subnetwork $A$ (or $B$ or $C$ ). All infected individuals and infected vectors may be recovered and become susceptible again. Without loss of generality, we set the recovery rates of all subnetworks and vectors be one. And, the three subnetworks and three vectors are SIS models.

Let $\rho_{k}(t), \eta_{l}(t)$ and $\xi_{m}(t)$ be the densities of infected nodes with degree $k, l$ and $m$ at time $t$ in subnetworks $A, B$ and $C$, respectively. Let $\vartheta_{1}, \vartheta_{2}$ and $\vartheta_{3}$ be the densities of the infective vectors $a, b$ and $c$ at time $t$, respectively. Here, $k=1,2, \cdots, n, l=1,2, \cdots, p$ and $m=1,2, \cdots, q$, where $n, p$ and $q$ are the number of max connections in subnetworks $A, B$ and $C$, respectively.

Then, the dynamical mean-field reaction rate equations of the whole network can be described as

$$
\left\{\begin{array}{l}
\dot{\rho}_{k}(t)=-\rho_{k}(t)+\left(1-\rho_{k}(t)\right)\left(\lambda_{1} k \Theta_{1}(t)+r_{32} \vartheta_{3}(t)\right), \\
\dot{\vartheta}_{1}(t)=-\vartheta_{1}(t)+r_{11} \rho(t)\left(1-\vartheta_{1}(t)\right), \\
\dot{\eta}_{l}(t)=-\eta_{l}(t)+\left(1-\eta_{l}(t)\right)\left(\lambda_{2} l \Theta_{2}(t)+r_{12} \vartheta_{1}(t)\right), \\
\dot{\vartheta}_{2}(t)=-\vartheta_{2}(t)+r_{21} \eta(t)\left(1-\vartheta_{2}(t)\right), \\
\dot{\xi}_{m}(t)=-\xi_{m}(t)+\left(1-\xi_{m}(t)\right)\left(\lambda_{3} m \Theta_{3}(t)+r_{22} \vartheta_{2}(t)\right), \\
\dot{\vartheta}_{3}(t)=-\vartheta_{3}(t)+r_{31} \xi(t)\left(1-\vartheta_{3}(t)\right),
\end{array}\right.
$$

where $\rho(t)=\sum_{k=1}^{n} P_{1}(k) \rho_{k}(t), \eta(t)=\sum_{l=1}^{p} P_{2}(l) \eta_{l}(t)$ and $\xi(t)=\sum_{m=1}^{q} P_{3}(m) \xi_{m}(t)$ denote the global infection density in subnetworks $A, B$ and $C$, in which $P_{1}(k), P_{2}(l)$ and $P_{3}(m)$ are the degree distributions of three subnetworks $A, B$ and $C$, respectively. Because the degrees of nodes in the network are uncorrelated, $\Theta_{1}(t)$ (or $\Theta_{2}(t)$ or $\Theta_{3}(t)$ ) represents the probability of a randomly 
chosen link pointing to an infected node in subnetwork $A$ (or $B$ or $C$ ), which can be written as

$$
\Theta_{1}(t)=\frac{1}{\langle k\rangle} \sum_{k=1}^{n} k P_{1}(k) \rho_{k}(t), \Theta_{2}(t)=\frac{1}{\langle l\rangle} \sum_{l=1}^{p} l P_{2}(l) \eta_{l}(t), \Theta_{3}(t)=\frac{1}{\langle m\rangle} \sum_{m=1}^{q} m P_{3}(m) \xi_{m}(t),
$$

in which $\langle k\rangle=\sum_{k=1}^{n} k P_{1}(k),\langle l\rangle=\sum_{l=1}^{p} l P_{2}(l)$ and $\langle m\rangle=\sum_{m=1}^{q} m P_{3}(m)$ are the average degrees of subnetworks $A, B$ and $C$, respectively.

Let $\rho_{k}(t)=y_{k}(t)$ for $k=1,2, \cdots, n, \eta_{l}(t)=y_{n+l}(t)$ for $l=1,2, \cdots, p, \xi_{m}(t)=y_{n+p+m}(t)$ for $m=1,2, \cdots, q, \vartheta_{1}(t)=y_{n+p+q+1}(t), \vartheta_{2}(t)=y_{n+p+q+2}(t), \vartheta_{3}(t)=y_{n+p+q+3}(t)$, and

$$
\tilde{\Omega}=\left\{\left(y_{1}, y_{2}, \cdots, y_{n+p+q+3}\right) \mid 0 \leq y_{i} \leq 1, i=1,2, \cdots, n+p+q+3\right\} .
$$

According to [43], the basic reproduction number can be computed by $R_{0}=\rho\left(F V^{-1}\right)$. The next-generation matrix $\tilde{\Gamma}=F V^{-1}$ is transformed into

$$
\tilde{\Gamma}=\left(\begin{array}{ccccccccc}
\lambda_{1} \frac{\left\langle k^{2}\right\rangle}{\langle k\rangle} & \lambda_{1} r_{32} & 0 & 0 & 0 & 0 & 0 & 0 & 0 \\
0 & 0 & 0 & 0 & 0 & 0 & 0 & 0 & 1 \\
0 & 0 & \lambda_{2} \frac{\left\langle l^{2}\right\rangle}{\langle l\rangle} & \lambda_{2} r_{12} & 0 & 0 & 0 & 0 & 0 \\
0 & 0 & 0 & 0 & 0 & 0 & 1 & 0 & 0 \\
0 & 0 & 0 & 0 & \lambda_{3} \frac{\left\langle m^{2}\right\rangle}{\langle m\rangle} & \lambda_{3} r_{22} & 0 & 0 & 0 \\
0 & 0 & 0 & 0 & 0 & 0 & 0 & 1 & 0 \\
r_{11}\langle k\rangle & r_{11} r_{32} & 0 & 0 & 0 & 0 & 0 & 0 & 0 \\
0 & 0 & r_{21}\langle l\rangle & r_{21} r_{12} & 0 & 0 & 0 & 0 & 0 \\
0 & 0 & 0 & 0 & r_{31}\langle m\rangle & r_{31} r_{22} & 0 & 0 & 0
\end{array}\right) .
$$

So, the basic reproduction number of model $(3.1)$ is $R_{0}=\rho(\tilde{\Gamma})$. Because $\tilde{\Gamma}$ is a nonnegative matrix, we know $\tilde{\Gamma}$ has a positive eigenvalue equals to $R_{0}$. By Perron-Frobenius theorem, we also can estimate $R_{0}$ of the model. And,

Theorem 3.1 When $R_{0}<1$, the disease-free equilibrium $E_{1}=(0,0, \cdots, 0)$ of the model (3.1) is locally asymptotically stable, while when $R_{0}>1, E_{1}$ becomes unstable.

Next, we analysis the global dynamics of system (3.1). The following theorem is needed to guarantee the positivity and boundedness of the system.

Theorem 3.2 For the model (3.1), the set $\tilde{\Omega}$ is positively invariant.

Proof We will prove that if $y(0) \in \tilde{\Omega}$, then $y(t) \in \tilde{\Omega}$ for all $t>0$. Let $\partial \tilde{\Omega}_{1}=\left\{y \in \tilde{\Omega} \mid y_{i}=0\right.$ for some $i\}, \partial \tilde{\Omega}_{2}=\left\{y \in \tilde{\Omega} \mid y_{i}=1\right.$ for some $\left.i\right\}$, where $i=1,2, \cdots, n+p+q+3$, and the 'outer normals' be denoted as $\delta_{i}^{1}=(\overbrace{0, \cdots, 0,-1}^{i}, 0, \cdots, 0)$ and $\delta_{i}^{2}=(\overbrace{0, \cdots, 0,1}^{i}, 0, \cdots, 0)$.

For an arbitrary set $\Delta,[45]$ had proved that $\Delta$ is invariant for $\mathrm{d} x / \mathrm{d} t=f(x)$, if at each point $y$ on the boundary of $\Delta$, the vector $f(y)$ is tangent or pointing into the set. It is easy to apply this result here, we can obtain

$$
\left.\frac{\mathrm{d} y}{\mathrm{~d} t}\right|_{y_{i}=0} \cdot \delta_{i}^{1}=-\left(\frac{\lambda_{1} k}{\langle k\rangle} \sum_{k \neq i} k P_{1}(k) \rho_{k}+r_{32} \vartheta_{3}\right) \leq 0, i=1, \cdots, n,
$$




$$
\begin{aligned}
& \left.\frac{\mathrm{d} y}{\mathrm{~d} t}\right|_{y_{j}=0} \cdot \delta_{j}^{1}=-\left(\frac{\lambda_{2} l}{\langle l\rangle} \sum_{l \neq j} l P_{2}(l) \eta_{l}+r_{12} \vartheta_{1}\right) \leq 0, j=n+1, \cdots, n+p, \\
& \left.\frac{\mathrm{d} y}{\mathrm{~d} t}\right|_{y_{s}=0} \cdot \delta_{s}^{1}=-\left(\frac{\lambda_{3} m}{\langle m\rangle} \sum_{m \neq s} m P_{3}(m) \xi_{m}+r_{22} \vartheta_{2}\right) \leq 0, s=n+p+1, \cdots, n+p+q, \\
& \left.\frac{\mathrm{d} y}{\mathrm{~d} t}\right|_{y_{g}=0} \cdot \delta_{g}^{1}=-r_{11} \sum_{k=1}^{n} P_{1}(k) \rho_{k} \leq 0, g=n+p+q+1, \\
& \left.\frac{\mathrm{d} y}{\mathrm{~d} t}\right|_{y_{h}=0} \cdot \delta_{h}^{1}=-r_{21} \sum_{l=1}^{p} P_{2}(l) \eta_{l} \leq 0, h=n+p+q+2, \\
& \left.\frac{\mathrm{d} y}{\mathrm{~d} t}\right|_{y_{r}=0} \cdot \delta_{r}^{1}=-r_{31} \sum_{m=1}^{q} P_{3}(m) \xi_{m} \leq 0, r=n+p+q+3, \\
& \left.\frac{\mathrm{d} y}{\mathrm{~d} t}\right|_{y_{u}=1} \cdot \delta_{u}^{2}=-y_{u} \leq 0, u=1,2, \cdots, n+p+q+3 .
\end{aligned}
$$

Thus, any solution of the system that begins with $y \in \partial \tilde{\Omega}_{1} \bigcup \partial \tilde{\Omega}_{2}$ will stay inside $\tilde{\Omega}$.

Let $y=\left(y_{1}, y_{2}, \cdots, y_{n+p+q+3}\right) \in \tilde{\Omega}$ and $L(y)$ be a column vector, then model (3.1) can be rewritten as

$$
\frac{\mathrm{d} y}{\mathrm{~d} t}=A y+L(y)
$$

where $A y$ is the linear part of $y$, and $L(y)$ is the nonlinear part of $y$. For $L(y)$, the $k$-th component is $-y_{k}\left[k \lambda_{1} \Theta_{1}(y)+r_{32} y_{n+p+q+3}\right], k=1,2, \cdots, n$, the $l$-th component is $-y_{l}\left[l \lambda_{2} \Theta_{2}(y)+\right.$ $\left.r_{12} y_{n+p+q+1}\right], l=n+1, n+2, \cdots, n+p$, the $m$-th component is $-y_{m}\left[m \lambda_{3} \Theta_{3}(y)+r_{22} y_{n+p+q+2}\right]$, $m=n+p+1, n+p+2, \cdots, n+p+q$, the $(n+p+q+1)$-th component is $-y_{n+p+q+1} r_{11} X$, the $(n+p+q+2)$-th component is $-y_{n+p+q+2} r_{21} Y$, and the $(n+p+q+3)$-th component is $-y_{n+p+q+3} r_{31} Z$, in which $\Theta_{1}(y)=\langle k\rangle^{-1} \sum_{k=1}^{n} k P_{1}(k) y_{k}(t), \Theta_{2}(y)=\langle l\rangle^{-1} \sum_{l=1}^{p} l P_{2}(l) y_{n+l}(t)$, $\Theta_{3}(y)=\langle m\rangle^{-1} \sum_{m=1}^{q} m P_{3}(m) y_{n+p+m}(t), X(t)=\sum_{k=1}^{n} P_{1}(k) y_{k}(t), Y(t)=\sum_{l=1}^{p} P_{2}(l) y_{n+l}(t)$, and $Z(t)=\sum_{m=1}^{q} P_{3}(m) y_{n+p+m}(t)$.

Let $s(A)=\max _{1 \leq i \leq n+p+q+3} \operatorname{Re} \lambda_{i}$, in which $\operatorname{Re} \lambda_{i}$ denotes the real part of $\lambda_{i}$, and $\lambda_{i}$ is the eigenvalue of $A$ for $i=1,2, \cdots, n+p+q+3$.

Remark 3.3 $s(A)<0 \Longleftrightarrow R_{0}<1, s(A)>0 \Longleftrightarrow R_{0}>1$.

Moreover, the following lemma is also needed to study the global stability of the model.

Lemma 3.4 (see [46]) Consider the system

$$
\frac{\mathrm{d} y}{\mathrm{~d} t}=A y+L(y)
$$

where $A$ is an $n \times n$ matrix and $L(y)$ is continuously differentiable in a region $D \in R^{n}$. Assume that

(1) the compact convex set $C \subset D$ is positively invariant with respect to (3.4), and $0 \in C$;

(2) $\lim _{y \rightarrow 0}\|L(y)\| /\|y\|=0$

(3) there exist $r>0$ and a (real) eigenvector $\omega$ of $A^{T}$ such that $(\omega \cdot y) \geq r\|y\|$ for all $y \in C$;

(4) $(\omega \cdot L(y)) \leq 0$ for all $y \in C$;

(5) $y=0$ is the largest positively invariant set contained in $H=\{y \in C \mid(\omega \cdot L(y))=0\}$. Then, either $y=0$ is globally asymptotically stable in $C$, or for any $y_{0} \in C-\{\mathbf{0}\}$ the solution 
$\varphi\left(t, y_{0}\right)$ of (3.4) satisfies $\liminf _{t \rightarrow \infty}\left\|\varphi\left(t, y_{0}\right)\right\| \geq m$, where $m>0$ is independent of the initial value $y_{0}$. Moreover, there exists a constant solution of (3.4), $y=y^{*}, y^{*} \in C-\{\mathbf{0}\}$.

Then, we have the following result.

Theorem 3.5 For the disease transmission model (3.1), the disease-free equilibrium $E_{1}$ is globally asymptotically stable in $\tilde{\Omega}$ if $R_{0}<1$, otherwise, the model admits a unique positive solution $y^{*} \in \tilde{\Omega}-\{\mathbf{0}\}$ if $R_{0}>1$.

Proof First, we will confirm that system (3.3) meets all hypotheses of Lemma 3.4. Condition (1) is satisfied by letting $C=\tilde{\Omega}$. Conditions (2) and (4) are clearly satisfied. For condition (3), note that $A^{T}$ is irreducible and $a_{i j} \geq 0$ whenever $i \neq j$, then there exists a positive eigenvector $x=\left(x_{1}, x_{2}, \cdots, x_{g}\right)$ of $A^{T}$ and the eigenvalue is $s\left(A^{T}\right), g=n+p+q+3$. Let $x_{0}=\min _{i} x_{i}$, for $y \in \tilde{\Omega}$, we obtain $(x \cdot y) \geq x_{0} \sum_{i=1}^{g} y_{i} \geq x_{0}\left(\sum_{i=1}^{g} y_{i}^{2}\right)^{\frac{1}{2}}$. Hence, condition (3) is achieved by letting $r=x_{0}$. In order to verify condition (5), we set $G=\{y \in \tilde{\Omega} \mid(\omega \cdot L(y))<0\}$. If $y \in G$, then

$$
\begin{aligned}
& \sum_{i=1}^{n} x_{i} y_{i}\left[\lambda_{1} i \Theta_{1}(y)+r_{32} y_{n+p+q+3}\right]+\sum_{i=1}^{p} x_{n+i} y_{n+i}\left[\lambda_{2} i \Theta_{2}(y)+r_{12} y_{n+p+q+1}\right] \\
& +\sum_{i=1}^{q} x_{n+p+i} y_{n+p+i}\left[\lambda_{3} i \Theta_{3}(y)+r_{22} y_{n+p+q+2}\right]+x_{n+p+q+1} y_{n+p+q+1} \cdot r_{11} X \\
& +x_{n+p+q+2} y_{n+p+q+2} \cdot r_{21} Y+x_{n+p+q+3} y_{n+p+q+3} \cdot r_{31} Z=0 .
\end{aligned}
$$

Because of each term of the above equation is nonnegative, we have $y_{i}=0$ for all $i=$ $1,2, \cdots, n+p+q+3$. Therefore, the only invariant set of system (3.3) contained in $G$ is $y=0$, so condition (5) is satisfied. Thus, all hypotheses of Lemma 3.4 are satisfied.

Next, we will prove that if $R_{0}>1$, there is only one constant solution $y=y^{*}$ in $\tilde{\Omega}-\{\mathbf{0}\}$. Assume that $y=y^{*}$ and $y=z^{*}>0$ are two constant solutions of (3.3) in $\tilde{\Omega}-\{\mathbf{0}\}$. If $y^{*} \neq z^{*}$, then there is at least one $i_{0}$ such that $y_{i_{0}}^{*} \neq z_{i_{0}}^{*}$, in which $y_{i_{0}}^{*}\left(z_{i_{0}}^{*}\right)$ is the $i_{0^{-}}$-th component of the vector $y^{*}\left(z^{*}\right)$ for $i_{0} \in\{1,2, \cdots, n+p+q+3\}$. Without loss of generality, we assume that $y_{i_{0}}^{*}>z_{i_{0}}^{*}$ and $y_{i_{0}}^{*} / z_{i_{0}}^{*} \geq y_{i}^{*} / z_{i}^{*}$ for all $i=1,2, \cdots, n+p+q+3$ and $i \neq i_{0}$. Then, by plugging them into (3.3), we obtain

$-y_{i_{0}}^{*}+\left(1-y_{i_{0}}^{*}\right)\left[\lambda_{1} i_{0} \Theta_{1}\left(y^{*}\right)+r_{32} y_{n+p+q+3}^{*}\right]=-z_{i_{0}}^{*}+\left(1-z_{i_{0}}^{*}\right)\left[\lambda_{1} i_{0} \Theta_{1}\left(z^{*}\right)+r_{32} z_{n+p+q+3}^{*}\right]=0$.

By the equivalent reformulation, as a result

$-z_{i_{0}}^{*}+\left(1-y_{i_{0}}^{*}\right) \frac{z_{i_{0}}^{*}}{y_{i_{0}}^{*}}\left[\lambda_{1} i_{0} \Theta_{1}\left(y^{*}\right)+r_{32} y_{n+p+q+3}^{*}\right]=-z_{i_{0}}^{*}+\left(1-z_{i_{0}}^{*}\right)\left[\lambda_{1} i_{0} \Theta_{1}\left(z^{*}\right)+r_{32} z_{n+p+q+3}^{*}\right]=0$.

$\operatorname{But}\left(z_{i_{0}}^{*} / y_{i_{0}}^{*}\right) y_{i}^{*} \leq z_{i}^{*}$ for all $i$ and $1-y_{i_{0}}^{*}<1-z_{i_{0}}^{*}$, thus from the above equality we obtain

$$
\left(1-y_{i_{0}}^{*}\right) \frac{z_{i_{0}}^{*}}{y_{i_{0}}^{*}}\left[\lambda_{1} i_{0} \Theta_{1}\left(y^{*}\right)+r_{32} y_{n+p+q+3}^{*}\right]<\left(1-z_{i_{0}}^{*}\right)\left[\lambda_{1} i_{0} \Theta_{1}\left(z^{*}\right)+r_{32} z_{n+p+q+3}^{*}\right] .
$$

That's a contradiction. Thus, there is only one constant solution $y^{*}=\left(y_{1}^{*}, \cdots, y_{n+p+q+3}^{*}\right)$ in $\tilde{\Omega}-\{\mathbf{0}\}$. The proof is completed.

Theorem 3.6 If $R_{0}>1$, then the unique endemic equilibrium $y^{*}=\left(y_{1}^{*}, y_{2}^{*}, \cdots, y_{n+p+q+3}^{*}\right)$ of model (3.1) is globally attractive in $\tilde{\Omega}-\{\mathbf{0}\}$. 
Proof Now, we define the following functions $f: \tilde{\Omega} \rightarrow R$ and $F: \tilde{\Omega} \rightarrow R$, where $f(y)=\max _{i}\left(y_{i} / y_{i}^{*}\right)$ and $F(y)=\min _{i}\left(y_{i} / y_{i}^{*}\right)$ for $y \in \tilde{\Omega} . f(y)$ and $F(y)$ are continuous and their derivatives exist along solutions of (3.3). Let $y=y(t)$ be a solution of system (3.3), for a given $t_{0}$ and a small enough constant $\epsilon>0$, we assume that $f(y(t))=y_{i_{0}}(t) / y_{i_{0}}^{*}$ for $t \in\left[t_{0}, t_{0}+\epsilon\right]$, $1 \leq i_{0} \leq n+p+q+3$. Then,

$$
\left.f^{\prime}\right|_{(3.3)}\left(y\left(t_{0}\right)\right)=\frac{y_{i_{0}}^{\prime}\left(t_{0}\right)}{y_{i_{0}}^{*}}, t \in\left[t_{0}, t_{0}+\epsilon\right],
$$

where $f^{\prime}=\limsup _{h \rightarrow 0^{+}} \frac{f(y(t+h))-f(y(t))}{h}$.

From system (3.3) we obtain

$$
y_{i_{0}}^{*} \frac{y_{i_{0}}^{\prime}\left(t_{0}\right)}{y_{i_{0}}\left(t_{0}\right)}=-y_{i_{0}}^{*}+\left(1-y_{i_{0}}\left(t_{0}\right)\right) \frac{y_{i_{0}}^{*}}{y_{i_{0}}\left(t_{0}\right)}\left[\lambda_{1} i_{0} \Theta_{1}\left(y^{*}\right)+r_{32} y_{n+p+q+3}^{*}\right] .
$$

According to the definition of $f(y(t))$, it follows that

$$
\frac{y_{i_{0}}\left(t_{0}\right)}{y_{i_{0}}^{*}} \geq \frac{y_{i}\left(t_{0}\right)}{y_{i}^{*}}, i=1,2, \cdots, n+p+q+3 .
$$

Then, if $f\left(y\left(t_{0}\right)\right)>1$, we can obtain

$$
y_{i_{0}}^{*} \frac{y_{i_{0}}^{\prime}\left(t_{0}\right)}{y_{i_{0}}\left(t_{0}\right)}<-y_{i_{0}}^{*}+\left(1-y_{i_{0}}\left(t_{0}\right)\right)\left[\lambda_{1} i_{0} \Theta_{1}\left(y^{*}\right)+r_{32} y_{n+p+q+3}^{*}\right]=0 .
$$

Because $y_{i_{0}}^{*}>0$ and $y_{i_{0}}\left(t_{0}\right)>0$, from the above inequality we obtain $y_{i_{0}}^{\prime}\left(t_{0}\right)<0$. So, $f\left(y\left(t_{0}\right)\right)>$ 1 deduces $\left.f^{\prime}\right|_{(3.3)}\left(y\left(t_{0}\right)\right)<0$.

Similarly, we can testify if $f\left(y\left(t_{0}\right)\right)=1$, then $\left.f^{\prime}\right|_{(3.3)}\left(y\left(t_{0}\right)\right) \leq 0$. And if $f\left(y\left(t_{0}\right)\right)<1$, we obtain $\left.f^{\prime}\right|_{(3.3)}\left(y\left(t_{0}\right)\right)>0$. By the same method, it can be easily verified that if $F\left(y\left(t_{0}\right)\right)>$ 1 , then $\left.F^{\prime}\right|_{(3.3)}\left(y\left(t_{0}\right)\right)<0$; if $F\left(y\left(t_{0}\right)\right)=1$, then $\left.F^{\prime}\right|_{(3.3)}\left(y\left(t_{0}\right)\right) \geq 0$; if $F\left(y\left(t_{0}\right)\right)<1$, then $\left.F^{\prime}\right|_{(3.3)}\left(y\left(t_{0}\right)\right)>0$. Letting

$$
U(y)=\max \{f(y)-1,0\}, V(y)=\max \{1-F(y), 0\},
$$

which are continuous and nonnegative for $y \in \tilde{\Omega}$. Note that, for any $t>0$, there exist

$$
\left.U^{\prime}\right|_{(3.3)}(y(t)) \leq 0,\left.V^{\prime}\right|_{(3.3)}(y(t)) \leq 0 .
$$

Let $H_{U}=\left\{y \in \tilde{\Omega}\left|U^{\prime}\right|_{(3.3)}(y(t))=0\right\}, H_{V}=\left\{y \in \tilde{\Omega}\left|V^{\prime}\right|_{(3.3)}(y(t))=0\right\}$, then $H_{U}=\{y \mid 0 \leq$ $\left.y_{i} \leq y_{i}^{*}\right\}$ and $H_{V}=\left\{y \mid y_{i}^{*} \leq y_{i} \leq 1\right\} \cup\{\mathbf{0}\}$.

By the LaSalle invariance principle, any solution of system (3.3) begins with $\tilde{\Omega}$ will reach $H_{U} \cap H_{V}$, and $H_{U} \cap H_{V}=\left\{y_{i}^{*}\right\} \cup\{\mathbf{0}\}$. However, by Lemma 3.4, if $y(0) \neq 0$ and $R_{0}>1$, we have $\liminf _{t \rightarrow \infty}\left\|\varphi\left(t, y_{0}\right)\right\| \geq m>0$. Then, if $y(0) \in \tilde{\Omega}-\{\mathbf{0}\}$ and $R_{0}>1$, we obtain any solution of (3.3) satisfies $\lim _{t \rightarrow \infty} y(t)=y^{*}$, so $y=y^{*}$ is globally attractive in $\tilde{\Omega}-\{\mathbf{0}\}$.

\section{Numerical Simulations}

In this section, we present some numerical examples to illustrate and complement our theoretical results and further explore the transmission dynamics. 


\subsection{One-way Circular-coupled Network}

In order to study the influence of interactions between subnetworks on epidemic spread in the complex network, we present abundant numerical simulations on system (2.1). Suppose that the joint degree distributions are independent, so we consider two kinds of networks, scale-free network and random network [47]. The scale-free network is a heterogeneous network, in which the degree distribution is $P(k)=k^{-\gamma} / \sum_{k} k^{-\gamma}$, the maximum degree $n \approx k_{0} \sqrt[\gamma-1]{N}$, where $N$ is the number of total nodes, $k_{0}$ is the minimum degree of the network. The random network is homogeneous, whose degree distribution is Poisson $P(k)=\lambda^{k} \mathrm{e}^{-\lambda} / k$ ! where constant $\lambda$ denotes the average degree. In the simulations, $\mathrm{A}, \mathrm{B}$ and $\mathrm{C}$ denote the inner contact patterns in subnetworks $A, B$ and $C$, respectively. Moreover, $\mathrm{AB}$ denotes the cross contact pattern in subnetwork $A$ connecting to $B$, and contact patterns $\mathrm{BC}$ and $\mathrm{CA}$ are similar. Let $\rho^{A}(t)=\sum_{i=0}^{n_{11}} \sum_{j=0}^{n_{12}} \sum_{k=0}^{n_{13}} P_{A}(i, j, k) \rho_{i, j, k}^{A}(t), \quad \rho^{B}(t)=\sum_{i=0}^{n_{21}} \sum_{j=0}^{n_{22}} \sum_{k=0}^{n_{23}} P_{B}(i, j, k) \rho_{i, j, k}^{B}(t)$, $\rho^{C}(t)=\sum_{i=0}^{n_{31}} \sum_{j=0}^{n_{32}} \sum_{k=0}^{n_{33}} P_{C}(i, j, k) \rho_{i, j, k}^{C}(t)$ as the corresponding total infected densities.

In the simulations, we set $N^{A}=N^{B}=N^{C}=1000, k_{0}=1, \gamma=2.8$, and $\mu_{1}=\mu_{2}=\mu_{3}=1$. First, we testify the availability of basic reproduction number $R_{0}$ obtained by matrix (2.5). In Fig. 3(1), all the infection rates are fixed at 0.15 , and $R_{0}=0.67<1$, we can see the disease in all subnetworks disappear in the end. In Fig. $3(2)$, all the infection rates are fixed at 0.28 , and $R_{0}=1.26>1$, the disease spreads and becomes endemic. In Fig. 3, all the contact patterns are scale-free. One can see that the values of $R_{0}$ are consistent with theoretical results very well.
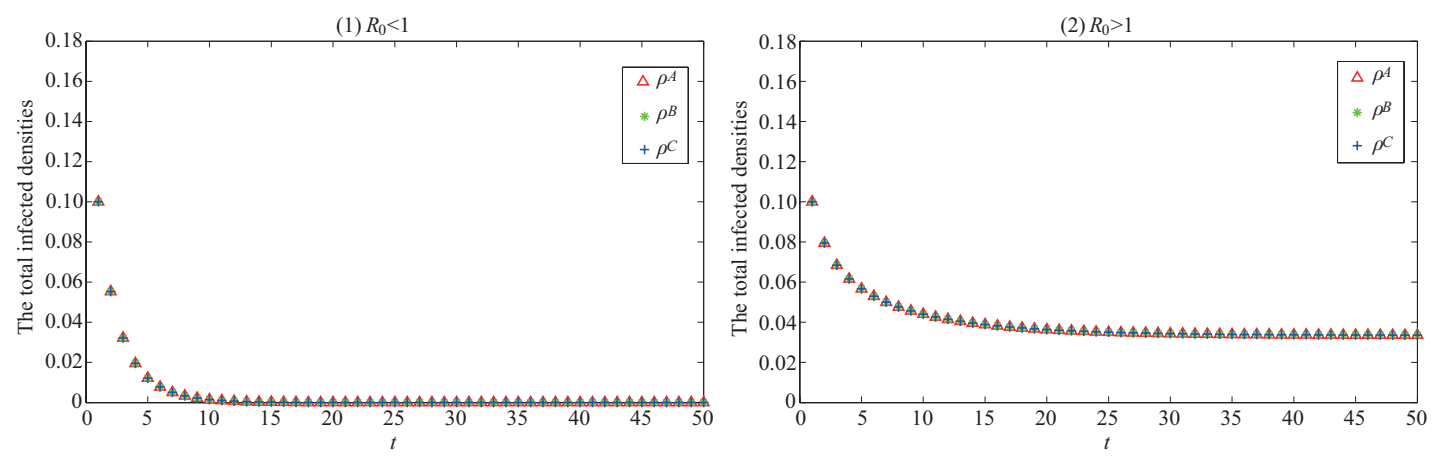

Figure 3 The changes of the total infected densities with respect to time $t$.

(1) $R_{0}=0.67<1 ;(2) R_{0}=1.26>1$

According to Fig. 4, we analysis the relationship between $R_{0}$ and the network size for different network structures. Hence, all the infection rates are 0.1, $\gamma=2.8$ in Fig. 4(1)-(2), $\gamma=2.6$ in Fig. 4(3)-(4), and other parameters be same as above. All contact patterns in the network admit the same average degree. Form the figure, we can observe that a scale-free subnetwork can make $R_{0}$ increase rapidly with the growth of network size, while a random subnetwork almost do not effect on $R_{0}$. Moreover, under the same cross contact patterns, subnetworks $A, B$ and $C$ have almost the same impact on $R_{0}$. From Fig. 4(3)-(4), one can see that the larger average degrees of contact patterns can yield the larger basic reproduction number of the network. Especially, the different network structures of cross contact patterns $\mathrm{AB}, \mathrm{BC}$ and $\mathrm{CA}$ hardly have any remarkable impact on $R_{0}$. 

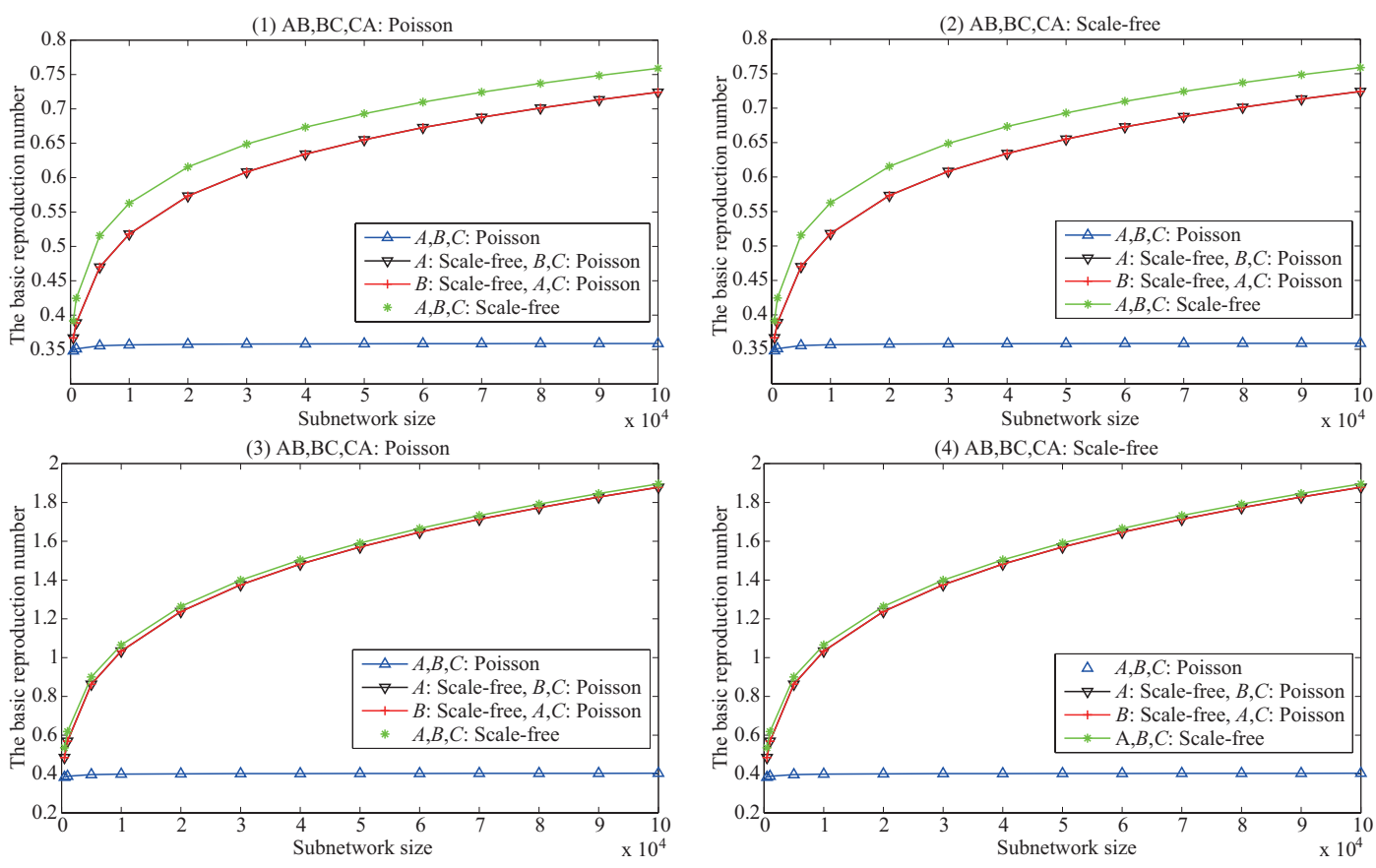

Figure 4 The relationship between the basic reproduction number and the network size for different network structures. (1)(2) $\gamma=2.8 ;(3)(4) \gamma=2.6$

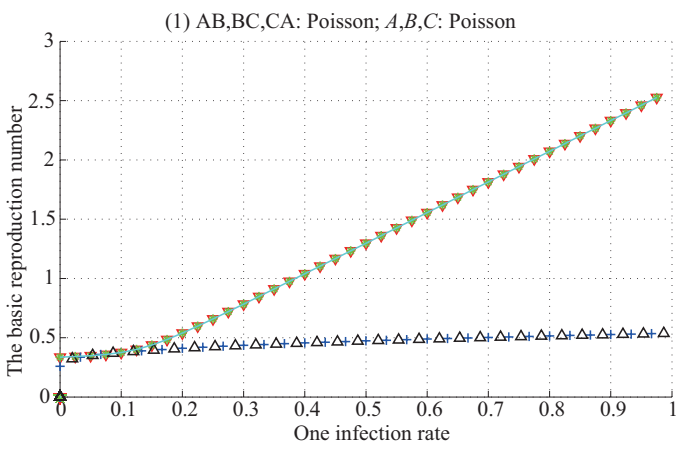

(3) AB,BC,CA: Poisson; $A, C$ : Scale-free, $B$ : Poisson

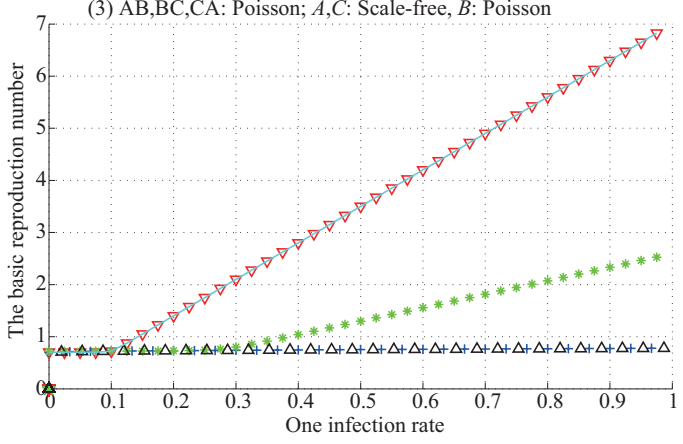

(2) $\mathrm{AB}, \mathrm{BC}, \mathrm{CA}:$ Scale-free; $A, B, C:$ Scale-free

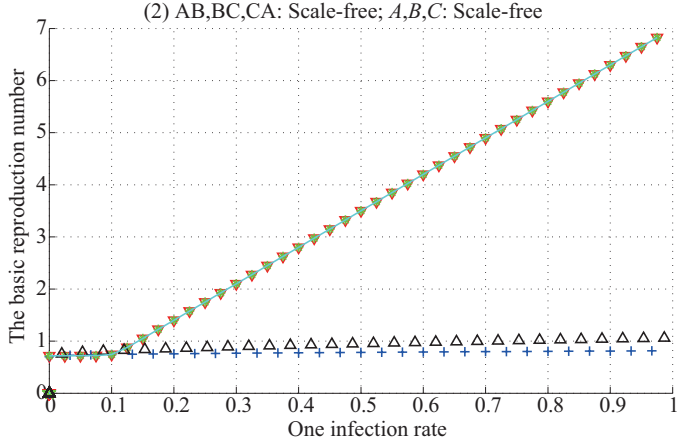

(4) AB,BC,CA: Scale-free; $A, C$ : Scale-free, $B$ : Poisson

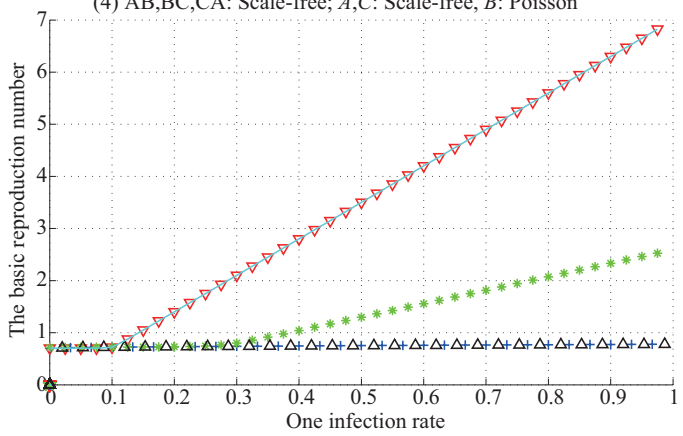

Figure 5 The relationship between the basic reproduction number and the infection rates for different network structures

Then, we continue studying how the infection rates affect $R_{0}$ on different network structures. In Fig. 5, we set $N^{A}=N^{B}=N^{C}=10000$, when one of the infection rates changes, the others are fixed at 0.1 . The other parameters be same as above. The label on the $\mathrm{x}$-axis in 
$\lambda_{11}, \lambda_{12}, \lambda_{22}, \lambda_{31}$ and $\lambda_{33}$ with the corresponding red $\nabla$, blue + , green $*$, black $\Delta$ and cyan - , respectively. All the contact patterns admit the same average degree. One can see that the internal infection rates $\lambda_{11}, \lambda_{22}$ and $\lambda_{33}$ have the same influence on $R_{0}$ under the same contact pattern, as well as the cross infection rates $\lambda_{12}$ and $\lambda_{31}$, and the internal infection rates have greater influence on $R_{0}$ than cross infection rates. Thus, the internal infection can affect the epidemic spreading more than the cross infection. If one of the inner contact patterns is scale-free, then $R_{0}$ increases quickly with the growth of inner infection rate.

Finally, we also set $N^{A}=N^{B}=N^{C}=1000, k_{0}=1, \gamma=2.8$, and $\mu_{1}=\mu_{2}=\mu_{3}=1$ in Fig. 6, when one of the infection rates changes, the others are fixed at 0.1. One can observe that the scale-free contact pattern can make the bigger total infected densities and the smaller epidemic thresholds of the network than the random contact pattern. With the inner infection rate $\lambda_{11}$ increases, the total infected density of subnetwork $A$ increases rapidly, followed by the infected density of subnetwork $B$, but almost no reaction with subnetwork $C$. The situations are similar when the increase of infection rates $\lambda_{22}$ and $\lambda_{33}$.
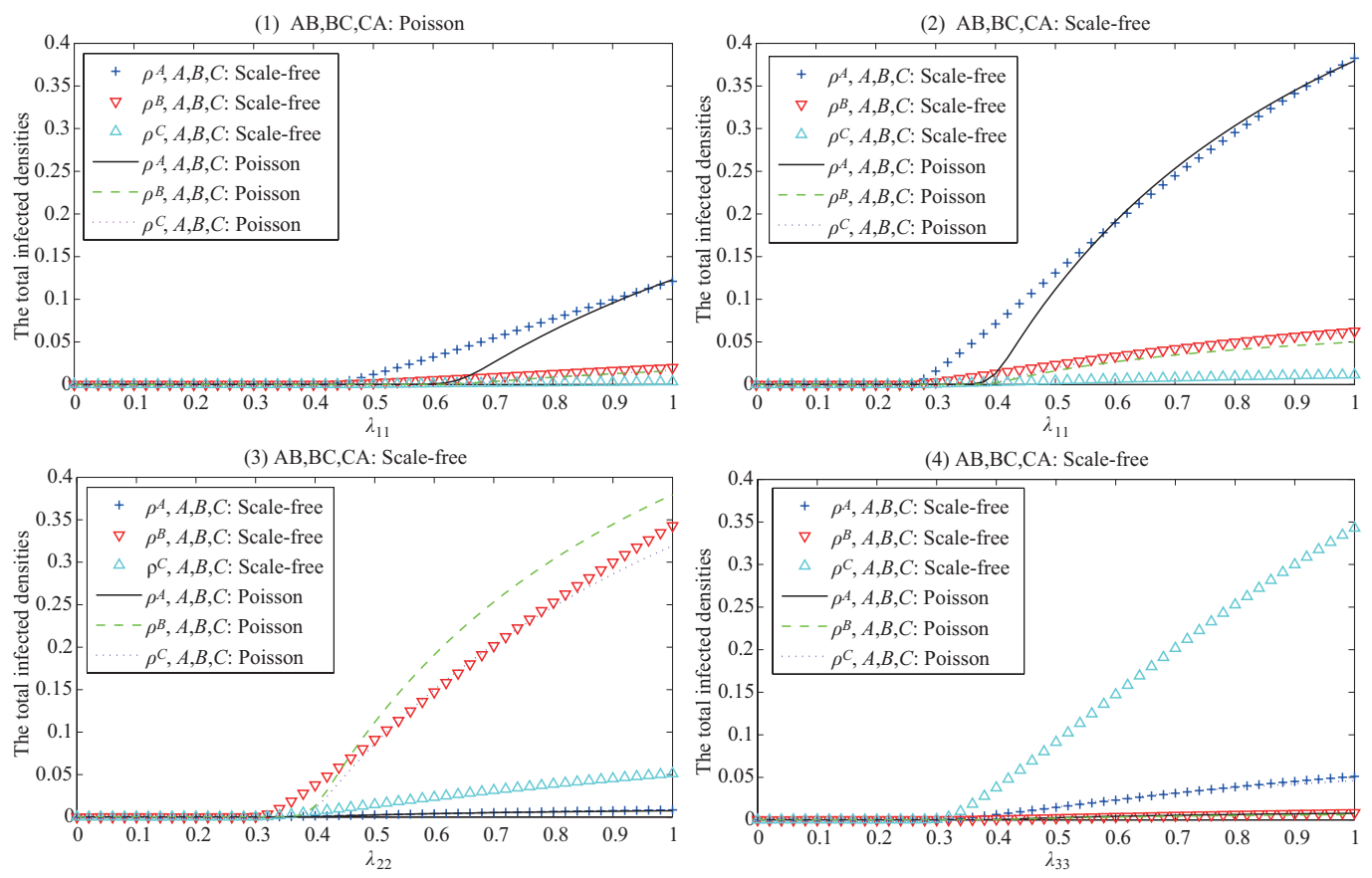

Figure 6 The total infected densities with respect to the infection rates and the contact patterns.

When one of the infection rates changes, the others are fixed at 0.1

\subsection{One-way Circular-coupled Network with Infective Media}

For the system (3.1), the contact pattern of human network is heterogeneous, while animal's contact pattern is homogeneous. Suppose that there are two human subnetworks and an animal subnetwork in the network. So, we set BA scale-free and ER random network as the topological structure of human network and animal network, respectively. Let $N^{A}=N^{B}=N^{C}=1000$, subnetworks $A$ and $B$ are BA scale-free networks and subnetwork $C$ is ER random network, and the same average degrees $\langle k\rangle=\langle l\rangle=\langle m\rangle=4$.

From Fig. 7, one can see that the influence of various infection rates on the basic reproduction number $R_{0}$. The line $+, \triangle, \nabla,-, *$ and $\times$ denotes the change of $\lambda_{1}, r_{32}, \lambda_{2}, r_{11}, \lambda_{3}$ 
and $r_{12}$, respectively. It is found that $\lambda_{2}$ contributes most to the basic reproduction number, $\lambda_{1}$ counts second, $\lambda_{3}$ counts third, and $r_{11}, r_{12}$ and $r_{32}$ have hardly impact on it.

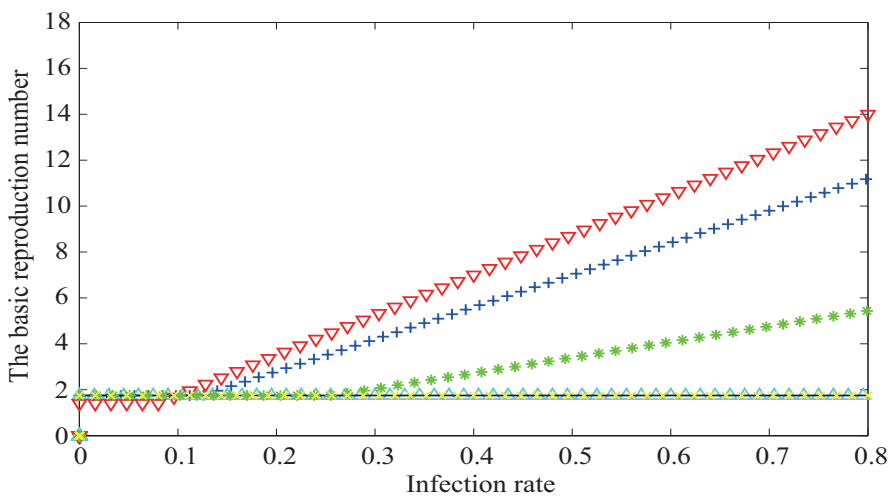

Figure 7 The influence of various infection rates on the basic reproduction number $R_{0}$.

When one of the infection rates changes, the others are fixed to be 0.1

Next, Fig. 8 presents the influence of infection rates on the stable infected density. We set $r_{11}=r_{12}=r_{21}=r_{22}=r_{31}=r_{32}=0.1$. The inner infection rates $\lambda_{2}=\lambda_{3}=0.2 \mathrm{in} \mathrm{left}$ subgraph and $\lambda_{1}=\lambda_{3}=0.2$ in right subgraph, respectively. From left subgraph, it is observed that the thresholds admit the same values for these populations, with the increases of infection rate $\lambda_{1}$, the infected density $\rho$ of subnetwork $A$ increases rapidly, more slowly in infected density $\eta$ and $\vartheta_{1}$, but almost no reaction in $\xi, \vartheta_{2}$ and $\vartheta_{3}$. Because the direction of epidemic spread between population networks and vectors is one-way, subnetwork $A$ almost has no influence on subnetwork $C$, vectors $b$ and $c$. The right subgraph is similar to the left.
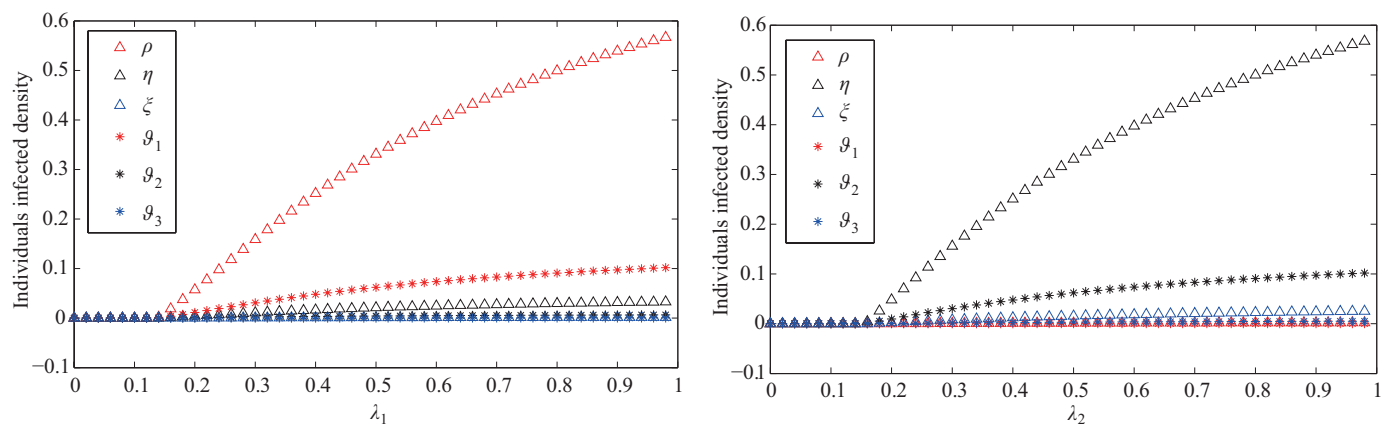

Figure 8 The relationship between individuals infected density and infection rate $\lambda_{1}\left(\lambda_{2}\right)$.

In the left subgraph, $\lambda_{2}=\lambda_{3}=0.2$. In the right subgraph, $\lambda_{1}=\lambda_{3}=0.2$

The time evolution of the disease spreading are described in Fig. 9-10. In left subgraph of Fig. 9, we set $\lambda_{1}=\lambda_{2}=\lambda_{3}=0.15$, and the initial values of three subnetworks are different. After a process of slow propagation, the infected density in subnetwork $A$ grows rapidly, the infected density in subnetwork $B$ begins to drop, then increases and reaches the stable state, but the infected density in subnetwork $C$ gradually becomes a small stable state, which be attribute to inner infections of subnetworks $A$ and $B$ as the dominant roles. Infected densities of all the vectors reach the lower stable states. In right subgraph of Fig. 9, we consider the disease just spreads by the vectors, that is, $\lambda_{1}=\lambda_{2}=\lambda_{3}=0$, and $R_{0}<1$, which implies the disease 
will disappear quickly. One can see that the diseases in subnetworks $B$ and $C$ are decrease rapidly, and even no incidence of subnetwork $A$. We set $\lambda_{1}=0.2, \lambda_{2}=\lambda_{3}=0$ in Fig. 10(1), which means the inner infection rate only in subnetwork $A$. The disease in $A$ increases rapidly and becomes stable, the incidences in $B$ and $C$ both reach a low stable state. Hence, we set $\lambda_{2}=0.2, \lambda_{1}=\lambda_{3}=0$ in (2) and $\lambda_{3}=0.25, \lambda_{1}=\lambda_{2}=0$ in (3), the conclusions are similar to subgraph (1). In this case, the infections among three subnetworks all play a important role in the propagation process, due to the particularity of transmission mode, the disease in one subnetwork has the bigger impact on the next vector, followed by the next subnetwork and almost no impact on another subnetwork.
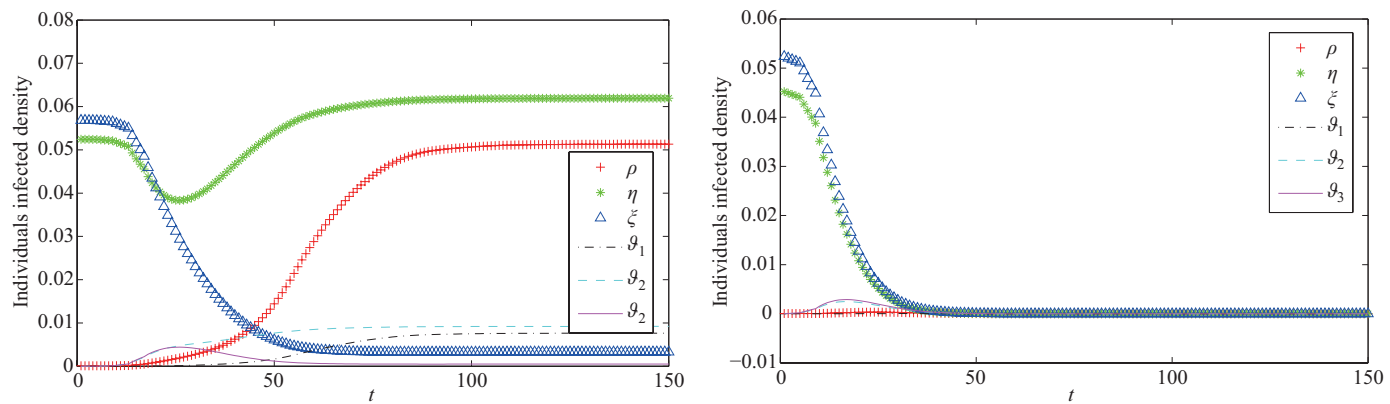

Figure 9 The changes of the infected densities with respect to time $t$, where $r_{11}=r_{12}=r_{21}=r_{22}=r_{31}=r_{32}=0.15$. In the left subgraph, $R_{0}=1.5$. In the right subgraph, $R_{0}=0.15$
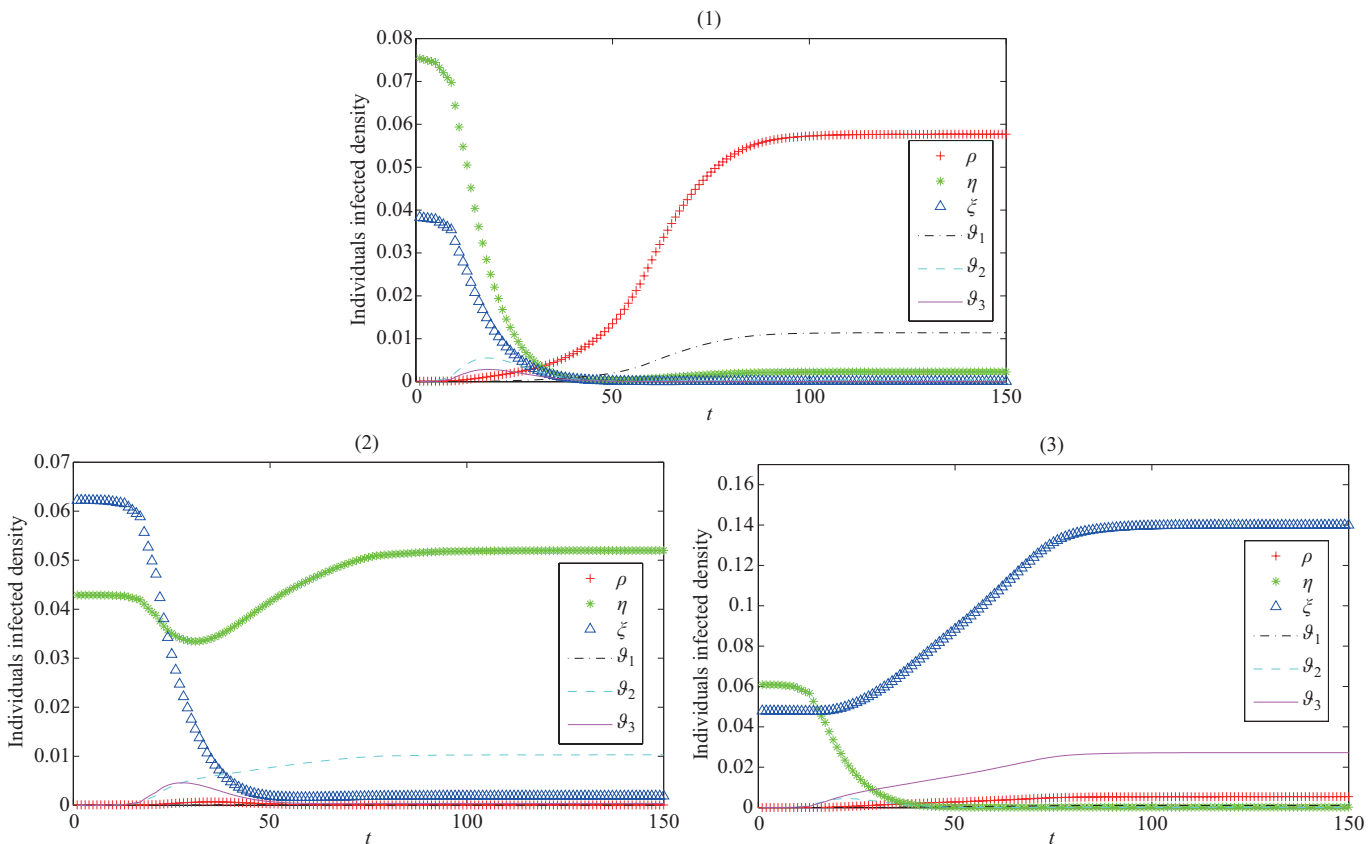

Figure 10 The changes of the infected densities with respect to time $t$, where $r_{11}=r_{12}=r_{21}=r_{22}=r_{31}=r_{32}=0.2$. (1) when $\lambda_{1}=0.2$ and $\lambda_{2}=\lambda_{3}=0$, then $R_{0}=2$; (2) when $\lambda_{2}=0.2$ and $\lambda_{1}=\lambda_{3}=0$, then $R_{0}=2 ;(3)$ when $\lambda_{3}=0.25$ and $\lambda_{1}=\lambda_{2}=0$, then $R_{0}=1.25$

Finally, Fig. 11 shows the stable infected density in subnetwork $A$ as a function of the transmission coefficients. If the inner infection rate $\lambda_{1}$ in subnetwork $A$ is fixed, then the 
infection from vector $c$ almost no effect on subnetwork $A$. When the infection rate $\lambda_{2}$ is fixed, the infected density $\rho$ increases with the growth of $\lambda_{1}$. If the infection rates $\lambda_{2}$ and $\lambda_{3}$ are very strong, however, the stable infected density of subnetwork $A$ is very small. Thus, $\lambda_{2}$ and $\lambda_{3}$ generate less incidence on the disease transmission than $\lambda_{1}$. The numerical simulations in accordance with the theoretical results, and independent of the initial values.
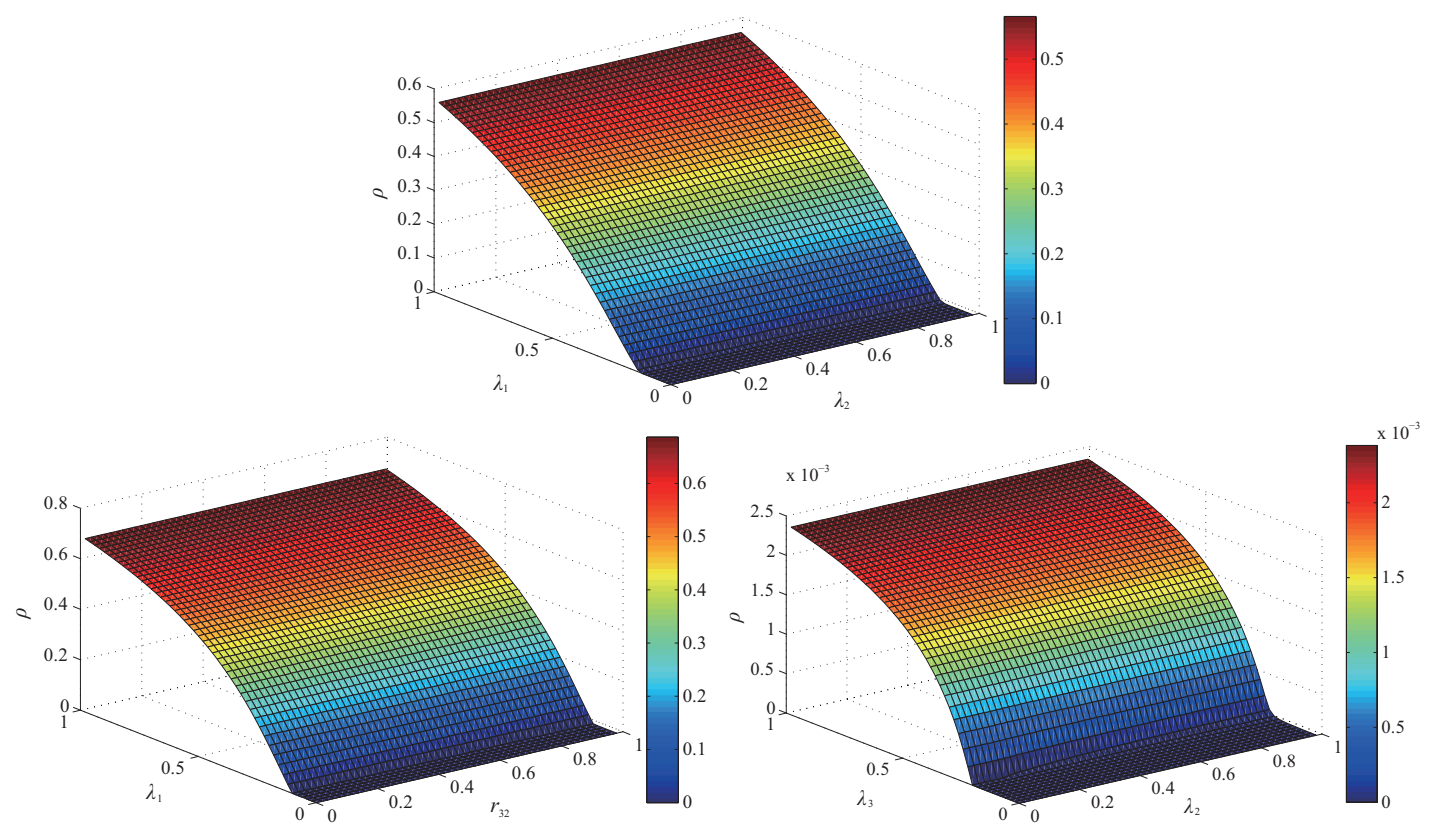

Figure 11 The relationship between the stable infected density of subnetwork $A$ and the transmission coefficients. When the parameters change, other infection rates are fixed at 0.05

\section{Conclusions and Discussions}

In this article, to study epidemic spread on three different populations with different contact patterns and infection rates, and zoonotic infections spread on directed interconnected networks, we established and investigated two one-way three-layer circular interdependent networks. The first epidemic model contains three different populations, and the direction of disease transmission is one-way, forming a circular-coupled network. Three different infective media are embedded in the second model. By Perron-Frobenius theorem, we estimate the basic reproduction number $R_{0}$ of the two models, then present global stability analysis of the disease-free and endemic equilibria of the first model. For the second model, the disease-free equilibrium is globally asymptotically stable if $R_{0}<1$, while the unique endemic equilibrium is globally attractive if $R_{0}>1$.

By numerical simulations, we found that both the inner and cross interactions can contribute to the epidemic spread, however, for the first model, the inner contact patterns have a bigger impact on $R_{0}$ than the cross contact patterns. Under the same contact pattern, the internal infection rates have greater influence on $R_{0}$ than the cross infection rates. Moreover, three subnetworks have almost the same impact on $R_{0}$, and the different cross contact patterns hardly have any remarkable impact on $R_{0}$. As a general situation, we set two human subnet- 
works and one animal subnetwork in the second model. Due to the particularity of transmission mode, with the increases of infection rate $\lambda_{1}$, subnetwork $A$ has the bigger impact on vector $a$, the smaller effect on subnetwork $B$, and almost no influence on subnetwork $C$, vectors $b$ and $c$, the others are similar. One can see that if the disease spreads in a subnetwork with a small infection rate, then the infection from the infected vector has almost no influence on the subnetwork. Based on these studies, we can find ways to effectively control the spread of diseases in interdependent networks. Hence, this work presents two special unidirectional circular-coupled epidemic models for better understanding the epidemic dynamics on interconnected networks in real life. Moreover, we hope our results may make a contribution to the practical applications to the control of zoonotic infectious diseases.

\section{References}

[1] Morens D M, Folkers G K, Fauci A S. Emerging infections: A perpetual challenge. Lancet Infect Dis, 2008, 8(11): $710-719$

[2] Peiris J S M, Chu C M, Cheng V C C, et al. Clinical progression and viral load in a community outbreak of coronavirus-associated SARS pneumonia: A prospective study. The Lancet, 2003, 361(9371): 1767-1772

[3] Jin Z, Ma Z E, Han M A. Global stability of an SIRS epidemic model with delays. Acta Math Sci, 2006, 26B(2): 291-306

[4] Kermack W O, Mckendrick A G. A contribution to the mathematical theory of epidemics. Proc R Soc A, 1927, 115: 700-721

[5] Watts D J, Strogatz S H. Collective dynamics of 'small-world' networks. Nature, 1998, 393: 440-442

[6] Barabási A L, Albert R. Emergence of scaling in random networks. Science, 1999, 286(5439): 509-512

[7] d'Onofrio A. A note on the global behavior of the network-based SIS epidemic model. Nonlinear Anal: Real World Appl, 2008, 9(4): 1567-1572

[8] Moreno Y, Pastor-Satorras R, Vespignani A. Epidemic outbreaks in complex heterogeneous networks. Eur Phys J B, 2002, 26(4): 521-529

[9] Pastor-Satorras R, Vespignani A. Epidemic dynamics and endemic states in complex networks. Phys Rev E, 2001, 63(6): 066117

[10] Pastor-Satorras R, Vespignani A. Epidemic dynamics in finite size scale-free networks. Phys Rev E, 2002, 65(3): 035108

[11] Wu Q C, Fu X C, Small M, Xu X J. The impact of awareness on epidemic spreading in networks. Chaos, 2012, 22: 013101

[12] Keeling M. The implications of network structure for epidemic dynamics. Theor Popul Biol, 2005, 67(1): $1-8$

[13] Fu X C, Small M, Walker D M, Zhang H F. Epidemic dynamics on scale-free networks with piecewise linear infectivity and immunization. Phys Rev E, 2008, 77(3): 036113

[14] Boguñá M, Castellano C, Pastor-Satorras R. Nature of the epidemic threshold for the susceptible-infectedsusceptible dynamics in networks. Phys Rev Lett, 2013, 111(6): 068701

[15] Peng X L, Xu X J, Fu X C, Zhou T. Vaccination intervention on epidemic dynamics in networks. Phys Rev E, 2013, 87(2): 022813

[16] Dharmaweera M N, Parthiban R, Sekercioglu Y A. Toward a power-efficient backbone network: The state of research. IEEE Commun Surv Tut, 2015, 17(1): 198-227

[17] Vidal M, Cusick M E, Barabási A L. Interactome networks and human disease. Cell, 2011, 144(6): 986-995

[18] Watts D J. A 21st century science. Nature, 2007, 445(7127): 489

[19] Gómez S, Diaz-Guilera A, Gómez-Gardeñes J, et al. Diffusion dynamics on multiplex networks. Phys Rev Lett, 2013, 110(8): 028701

[20] Solé-Ribalta A, De Domenico M, Kouvaris N E, et al. Spectral properties of the Laplacian of multiplex networks. Phys Rev E, 2013, 88(3): 032807

[21] De Domenico M, Granell C, Porter M A, et al. The physics of spreading processes in multilayer networks. Nat Phys, 2016, 12: 901-906 
[22] Boccaletti S, Bianconi G, Criado R, et al. The structure and dynamics of multilayer networks. Phys Reps, 2014, 544(1): 1-122

[23] Arbi A, Cherif F, Aouiti C, Touati A. Dynamics of new class of hopfield neural netwarks with time-varying and distributed delays. Acta Math Sci, 2016, 36B(3): 891-912

[24] Granell C, Gómez S, Arenas A. Dynamical interplay between awareness and epidemic spreading in multiplex networks. Phys Rev Lett, 2013, 111(12): 128701

[25] Saumell-Mendiola A, Serrano M A, Boguñá M. Epidemic spreading on interconnected networks. Phys Rev E, 2012, 86(2): 026106

[26] Bonaccorsi S, Ottaviano S, Pellegrini F D, Socievole A, Mieghem P V. Epidemic outbreaks in two-scale community networks. Phys Rev E, 2014, 90(1): 012810

[27] Son S W, Bizhani G, Christensen C, Grassberger P. Percolation theory on interdependent networks based on epidemic spreading. Europhys Lett, 2012, 97(1): 16006

[28] Zhao D W, Li L X, Peng H P, Luo Q, Yang Y X. Multiple routes transmitted epidemics on multiplex networks. Phys Lett A, 2014, 378(10): 770-776

[29] Wang Z, Andrews M A, Wu Z X, Wang L, Bauch C T. Coupled disease-behavior dynamics on complex networks: A review. Phys Life Rev, 2015, 15: 1-29

[30] Khanafer A, Basar T, Gharesifard B. Stability of epidemic models over directed graphs: A positive systems approach. Automatica, 2016, 74: 126-134

[31] Wang L N, Sun M F, Chen S S, Fu X C. Epidemic spreading on one-way-coupled networks. Phys A, 2016, 457: $280-288$

[32] Ogden N H, Bigras-Poulin M, O'Callaghan C J, et al. A dynamic population model to investigate effects of climate on geographic range and seasonality of the tick Ixodes scapularis. Int J Parasitol, 2005, 35(4): 375-389

[33] Wu X T, Duvvuri V R, Lou Y J, et al. Developing a temperature-driven map of the basic reproductive number of the emerging tick vector of Lyme disease Ixodes scapularis in Canada. J Theor Biol, 2013, 319: $50-61$

[34] Caraco T, Gardner G, Maniatty W, Deelman E, Szymanski B K. Lyme disease: Self-regulation and pathogen invasion. J Theor Biol, 1998, 193: 561-575

[35] Lou Y J, Wu J H, Wu X T. Impact of biodiversity and seasonality on Lyme-pathogen transmission. Theor Biol Med Model, 2014, 11: 50

[36] Lou Y J, Wu J H. Tick seeking assumptions and their implications for Lyme disease predictions. Ecol Compl, 2014, 17: 99-106

[37] Wolfe N D, Dunavan C P, Diamond J. Origins of major human infectious diseases. Nature, 2007, 447: 279-283

[38] Kieny M P, Lathe R, Drillien R, et al. Expression of rabies virus glycoprotein from a recombinant vaccinia virus. Nature, 1984, 312: 163-166

[39] Shi H J, Duan Z S, Chen G R. An SIS model with infective medium on complex networks. Phys A, 2008, 387(8/9): 2133-2144

[40] Wang Y, Jin Z, Yang Z M, et al. Global analysis of an SIS model with infective vector on complex networks. Nonlinear Anal: Real World Appl, 2011, 13(2): 543-557

[41] Xia C Y, Wang L, Sun S W, Wang J. An SIR model with infection delay and propagation vector in complex networks. Nonlinear Dyn, 2012, 69(3): 927-934

[42] Zhu G H, Chen G R, Zhang H F, Fu X C. Propagation dynamics of an epidemic model with infective media connecting two separated networks of populations. Commun Nonlinear Sci Numer Simul, 2012, 69(3): 2588-2594

[43] Driessche P van den, Watmough J. Reproduction numbers and sub-threshold endemic equilibria for compartmental models of disease transmission. Math Biosci, 2002, 180(1/2): 29-48

[44] Zhao X Q, Jing Z J. Global asymptotic behavior in some cooperative systems of functional differential equations. Can Appl Math Q, 1996, 4(4): 421-444

[45] Yorke J A. Invariance for ordinary differential equations. Theory Comput Syst, 1967, 1(4): 353-372

[46] Lajmanovich A, Yorke J A. A deterministic model for gonorrhea in a nonhomogeneous population. Math Biosci, 1976, 28(3/4): 221-236

[47] Newman M E J. The structure and function of complex networks. SIAM Rev, 2003, 45(2): 167-256 